\title{
Analysis of Network Motifs in Cellular Regulation: Structural Similarities, Input-Output Relations and Signal Integration
}

\author{
Ronny Straube \\ Max Planck Institute for Dynamics of Complex Technical Systems Magdeburg, Sandtorstr. 1, D-39106 Magdeburg
}

\begin{abstract}
Much of the complexity of regulatory networks derives from the necessity to integrate multiple signals and to avoid malfunction due to cross-talk or harmful perturbations. Hence, one may expect that the input-output behavior of larger networks is not necessarily more complex than that of smaller network motifs which suggests that both can, under certain conditions, be described by similar equations. In this review, we illustrate this approach by discussing the similarities that exist in the steady state descriptions of a simple bimolecular reaction, covalent modification cycles and bacterial two-component systems. Interestingly, in all three systems fundamental input-output characteristics such as thresholds, ultrasensitivity or concentration robustness are described by structurally similar equations. Depending on the system the meaning of the parameters can differ ranging from protein concentrations and affinity constants to complex parameter combinations which allows for a quantitative understanding of signal integration in these systems. We argue that this approach may also be extended to larger regulatory networks.
\end{abstract}

Keywords: ultrasensitivity, substrate competition, cooperativity, covalent modification cycles, two-component systems

\section{Introduction}

Biological networks are complex - not just by the number of their components, but also by the number and specificity of their interactions. Despite increasing knowledge of the molecular keyplayers involved in specific regulatory systems and signaling pathways simulating larger networks does not necessarily lead to deeper insights. It has thus become a useful strategy to analyze smaller recurring network structures called network motifs. Since the seminal work of Milo et al. (Milo et al., 2002), which originally focused on gene regulatory networks, the systematic analysis of network motifs has led to an increasing list of circuits that may generate specific input-output behavior such as ultrasensitivity and thresholds (Goldbeter and Koshland Jr., 1981; Gunawardena, 2005; Gomez-Uribe et al., 2007), foldchange detection (Geontoro et al., 2009; Adler et al., 2014; Olsman and Geontoro, 2016), concentration robustness (Shinar et al., 2007, 2009; Shinar and Feinberg, 2010) or multistability (Ferrell, Jr., 2002; Markevich et al., 2004; Tiwari et al., 2011; Straube and Conradi, 2013). Using methods from control theory network motifs were also analyzed from an engineering perspective (Sauro and Kholodenko, 2004).

Despite this increasing knowledge it has remained challenging to predict the behavior of larger networks based on the known behavior of its constituent parts (Rosenfeld et al., 2007). Exceptions are multistationarity and certain forms of robustness which can be detected based on algebraic properties of a network (Craciun et al., 2006; Conradi et al., 2007; Shinar and Feinberg, 2010; Dexter et al., 2015; Sontag, 2017), i.e. without detailed knowledge of reaction rates and parameters. However, while these methods can be used to decide whether a given sys- tem has a certain property they often do not say much about the parameter range or the biological conditions under which a certain behavior occurs. Obtaining this type of information typically requires more detailed analysis of the governing equations which will be the strategy advocated in this review.

To this end, we shall focus on mass-action networks with at most bimolecular interactions. Starting with the most simple of such systems, the receptor-ligand binding motif, we will stepwise extend the analysis to more complex network motifs. First, we show how thresholds and ultrasensitivity can arise through cooperativity in ligand binding and substrate competition. Subsequently, we consider covalent modification cycles which represent the elementary building blocks for many eukaryotic signaling networks such as protein kinase cascades or multisite phosphorylation systems, which are reviewed elsewhere (Heinrich et al., 2002; Salazar and Höfer, 2009; Ferrell Jr. and Ha, 2014). Finally, we discuss regulatory properties of two-component systems which share some similarity with covalent modification cycles, but occur mostly in bacteria (Stock et al., 2000). Again, we focus on the most simple regulatory structures leaving out more complicated architectures such as phosphorelay systems (Tiwari et al., 2011).

A major goal of this review is to highlight structural similarities that exist between the steady state equations characterizing the input-output behavior of different network motifs. Despite the fact that the transient dynamics will, in general, be different for different systems it turns out that in certain limiting regimes the steady state behavior of the network motifs considered in this review can be characterized by one of the following three 
types of (quadratic) equations

$$
\begin{gathered}
x^{2}-(A+B+C) x+q A B=0 \\
x^{2}+(A+B-C) x-B C=0 \\
\alpha \frac{A-x}{B+A-x}=\frac{x}{C+x}
\end{gathered}
$$

where $x$ denotes the concentration for the quantity of interest while $A, B, C, q$ and $\alpha$ denote single parameters or parameter combinations depending on the system. The above three equations arise in the analysis of receptor-ligand binding (Eqs. 1 and 2) and covalent modification cycles (Eq. 3) which may, thus, be regarded as 'elementary' network motifs. While the focus of this review lies on small networks and network motifs the presented methods may also be applicable to larger networks.

\section{Receptor-Ligand Binding}

\subsection{The LR-type equation}

The reversible binding of a ligand to a receptor is described by the reaction (Fig. 1A)

$$
L+R \underset{k_{d}^{-}}{\stackrel{k_{d}^{+}}{\rightleftarrows}} L R
$$

where the dissociation constant $K_{d}=k_{d}^{-} / k_{d}^{+}$represents a measure for the binding affinity of the ligand. Assuming that the concentrations of ligand and receptor are contant, i.e.

$$
\begin{aligned}
& {[L]+[L R]=L_{T}} \\
& {[R]+[L R]=R_{T}}
\end{aligned}
$$

the dynamics of the receptor-ligand complex is described by the ordinary differential equation (ODE)

$$
\frac{d[L R]}{d t}=k_{d}^{+}\left(L_{T}-[L R]\right)\left(R_{T}-[L R]\right)-k_{d}^{-}[L R]
$$

where $L_{T}$ and $R_{T}$ denote the total concentrations of ligand and receptor, respectively. Eq. (5) is one of the few nonlinear equations arising in mathematical biology that is exactly solvable (Tzafriri, 2003). If, initially, the concentration of LR vanishes its solution is given by

$$
[L R](t)=[L R]_{-} \frac{1-e^{-t / \tau_{s}}}{1-\frac{[L R]_{-}}{[L R]_{+}} e^{-t / \tau_{s}}}
$$

where

$$
[L R]_{ \pm}=\frac{L_{T}+R_{T}+K_{d}}{2} \pm \sqrt{\frac{\left(L_{T}+R_{T}+K_{d}\right)^{2}}{4}-L_{T} R_{T}}
$$

are the solutions of the steady state equation $(d[L R] / d t=0)$

$$
[L R]^{2}-\left(L_{T}+R_{T}+K_{d}\right)[L R]+L_{T} R_{T}=0,
$$

and

$$
\tau_{s}=\frac{1}{k_{d}^{-}} \frac{K_{d}}{\sqrt{\left(L_{T}+R_{T}+K_{d}\right)^{2}-4 L_{T} R_{T}}}
$$

denotes the time scale on which the stable steady state is reached for $t \gg \tau_{s}$ (cf. Eq. 6). Due to the constraint $0 \leq[L R] \leq \min \left(L_{T}, R_{T}\right)$ the latter is given by $[L R]_{-}$. As shown below the quadratic equation (8) frequently arises in the analysis of network motifs, so we denote it by LR-type equation.

It is instructive to analyze the expressions for the steady state and the time scale $\tau_{s}$ in the limits of low and high affinity. For definiteness we consider the receptor concentration as fixed and the ligand concentration as variable. In that case, the highaffinity limit is defined by $K_{d} \ll R_{T}$ and the stimulus-response curve can be approximated by (Straube, 2015)

$$
[L R] \approx \begin{cases}L_{T}\left(1-\frac{K_{d}}{R_{T}-L_{T}}\right), & L_{T}<R_{T} \\ R_{T}\left(1-\frac{K_{d}}{L_{T}-R_{T}}\right), & L_{T}>R_{T}\end{cases}
$$

while the expression for $\tau_{s}$ simplifies to (cf. subsection 6.1)

$$
\tau_{s} \approx\left\{\begin{array}{ll}
\frac{1}{k_{d}^{-}} \frac{K_{d}}{\left(R_{T}-L_{T}\right)}, & L_{T}<R_{T} \\
\frac{1}{k_{d}^{-}} \frac{K_{d}}{\left(L_{T}-R_{T}\right)}, & L_{T}>R_{T}
\end{array} .\right.
$$

In the low-affinity limit, defined by $K_{d} \gg R_{T}$, the approximations read

$$
\begin{aligned}
{[L R] } & \approx \frac{L_{T} R_{T}}{K_{d}+L_{T}+R_{T}} \\
\tau_{s} & \approx \frac{1}{k_{d}^{-}} \frac{K_{d}}{K_{d}+L_{T}+R_{T}} .
\end{aligned}
$$

Hence, in the high-affinity limit the receptor behaves as a stoichiometric inhibitor since the concentration of the complex increases in 1:1 stoichiometry with the ligand concentration until saturation (Fig. 1B). In contrast, the time scale for reaching the steady state $\tau_{s}$ exhibits a non-monotonic dependence on the ligand concentration with a maximum at $L_{T}=R_{T}$ (Fig. 1D). The latter can be rationalized as follows: If there is a surplus of either reaction partner (i.e. $L_{T} \gg R_{T}$ or $R_{T} \gg L_{T}$ ) a steady state is quickly reached because the probability for any molecule of the low-abundant species to find a molecule from the high-abundant species is large during the whole course of the reaction in which the high-abundant species is rarely depleted. The situation is different when ligands and receptors are present in comparable amounts (i.e. $L_{T} \approx R_{T}$ ) because in the high-affinity regime essentially all ligands will be bound to receptors in steady state. Hence, as the reaction proceeds both ligand and receptor pools are depleted simultaneously, thereby lowering the reaction rate which increases the time scale for reaching the steady state. In fact, from Eq. (9) one can show that $\tau_{s} \sim \sqrt{K_{d} / R_{T}}$ when $L_{T}=R_{T}$ (cf. Section 6.1). In contrast, in the low-affinity regime [LR] increases hyperbolically with the ligand concentration while the time scale for reaching the steady state remains almost constant for $L_{T}<R_{T}$ and decreases monotonically for $L_{T}>R_{T}$ (Fig. 1C and E).

\subsection{Synthesis and degradation}

It is an interesting question how changing the boundary conditions affects the dynamic behavior of a system. In general, changing boundary conditions may not only affect the transient 

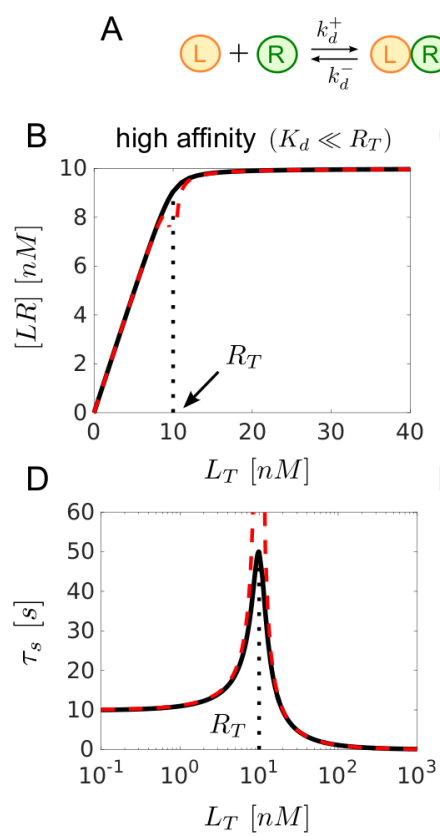

$K_{d}=\frac{k_{d}^{-}}{k_{d}^{+}}$

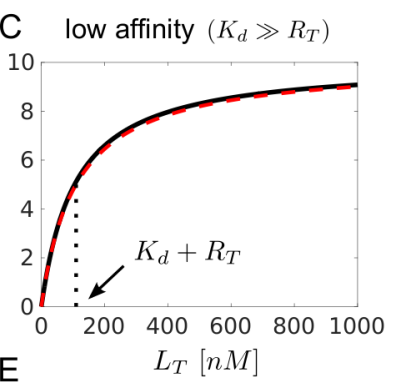

E

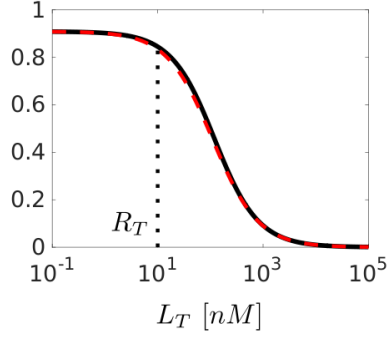

Figure 1: (A) Receptor-ligand binding motif. (B)-(E) Stimulus-response curves and time scales for reaching a new steady state in the strong and weak binding limits. Solid lines represent the exact solutions in Eq. (7) (B,C) and Eq. (9) $(\mathrm{D}, \mathrm{E})$ while the dashed lines denote the approximations in Eq. (10) (B), Eq. (12) (C), Eq. (11) (D) and Eq. (13) (E). Parameters: $k_{d}^{+}=0.01 /(n M \cdot s)$, $k_{d}^{-}=k_{d}^{+} \cdot K_{d}$ where $K_{d}=0.1 n M(\mathrm{~B}, \mathrm{D})$ and $K_{d}=100 n M(\mathrm{C}, \mathrm{E})$.

dynamics, but also the steady state behavior of a system. In the case of receptor-ligand binding Buchler and Louis have shown (Buchler and Louis, 2008) that going from a closed system with mass-conservation to an open system with constitutive synthesis for $\mathrm{L}$ and $\mathrm{R}$

$$
\emptyset \stackrel{k_{s, L}}{\rightarrow} L, \quad \emptyset \stackrel{k_{s, R}}{\rightarrow} R
$$

and linear degradation of the form

$$
L \stackrel{k_{d, L}}{\rightarrow} \emptyset, \quad R \stackrel{k_{d, R}}{\rightarrow} \emptyset, \quad L R \stackrel{k_{d, L R}}{\rightarrow} \emptyset
$$

leads again to a LR-type steady state equation for the receptorligand complex that is given by

$$
[L R]^{2}-\frac{k_{s, L}+k_{s, R}+\kappa}{k_{d, L R}}[L R]+\frac{k_{s, L} k_{s, R}}{k_{d, L R}^{2}}=0 .
$$

Note that Eq. (8) becomes identical with Eq. (14) through the substitution

$$
L_{T} \leftrightarrow \frac{k_{s, L}}{k_{d, L R}}, \quad R_{T} \leftrightarrow \frac{k_{s, R}}{k_{d, L R}}, \quad K_{d} \leftrightarrow \frac{\kappa}{k_{d, L R}} .
$$

Here, $\kappa=\left(k_{d, L} k_{d, R} / k_{d, L R}\right) \kappa_{D}$ is defined in terms of the in vivo dissociation constant $\kappa_{D}=\left(k_{d}^{-}+k_{d, L R}\right) / k_{d}^{+}$which is always larger than the in vitro dissociation constant $K_{d}=k_{d}^{-} / k_{d}^{+}$. For fixed receptor synthesis rate $k_{s, R}$ one may again define a highaffinity $\left(\kappa \ll k_{s, R}\right)$ and a low-affinity regime $\left(\kappa \gg k_{s, R}\right)$, and utilize the correspondence in Eq. (15) to derive approximate expressions similar to those in Eqs. (10) and (12).

\subsection{The L-type equation}

In many occasions the species of interest is the free form of the ligand rather than the receptor-ligand complex. The steady state equation for the free ligand can be obtained from that of the complex by substituting the conservation relation (4) into Eq. (8) which yields another type of quadratic equation

$$
[L]^{2}+\left(R_{T}+K_{d}-L_{T}\right)[L]-K_{d} L_{T}=0
$$

that we shall denote by L-type equation. Using the approximations for $[L R]$ and the conservation relation (4) one can readily derive corresponding approximations for $[L]$. In the highaffinity limit $\left(K_{d} \ll R_{T}\right)$ this yields

$$
[L] \approx\left\{\begin{array}{cc}
\frac{K_{d} L_{T}}{R_{T}-L_{T}}, & L_{T}<R_{T} \\
L_{T}-R_{T}+\frac{K_{d} R_{T}}{L_{T}-R_{T}}, & L_{T}>R_{T}
\end{array}\right.
$$

while the low-affinity approximation $\left(K_{d} \gg R_{T}\right)$ becomes

$$
[L] \approx L_{T} \frac{K_{d}+L_{T}}{K_{d}+L_{T}+R_{T}} \approx L_{T}
$$

From the expression in Eq. (17) we see that $[L]$ exhibits threshold behavior, i.e. below the threshold $L_{T}=R_{T}$ it remains low $\left(O\left(K_{d} / R_{T}\right)\right)$, and it increases linearly beyond the threshold.

This threshold-linear response behavior has been observed in diverse systems. It occurs, for example, in the context of molecular titration if the active form of a transcription factor is sequestered by a repressor into an inactive complex (Buchler and Louis, 2008) or if the translation of a mRNA is inhibited through sequestration of the target mRNA by a small repressor RNA (Levine et al., 2007). In the latter case Levine et al. derived the following steady state equation (Fig.2A)

$$
\left[m_{T}\right]^{2}+\left(\alpha_{R}+\lambda-\alpha_{T}\right) \frac{\left[m_{T}\right]}{\beta_{T}}-\frac{\alpha_{T} \lambda}{\beta_{T}^{2}}=0, \quad \lambda=\frac{\beta_{R} \beta_{T}}{k}
$$

which is structurally identical with the L-type equation (Eq. 16). Here, $\alpha_{R}$ and $\alpha_{T}$ denote the synthesis rates for repressor and target mRNA whereas $\lambda=\beta_{R} \beta_{T} / k$ is related to the mRNA turnover rates and the association rate (Fig. 2A). Similar as for the case of receptor-ligand binding in the 'high-affinity' regime $\lambda \ll \alpha_{R}$ the concentration of the target mRNA $\left[m_{T}\right]$ remains low until a threshold is crossed beyond which the mRNA level increases proportionally to the synthesis rate (Fig.2B).

\subsection{Approximation at the threshold}

In the high-affinity regime the approximations listed in Eqs. (10), (11) and (17) become singular at the threshold $\left(L_{T}=R_{T}\right)$ because the leading order of the exact solution of the quadratic equation becomes of $O(\sqrt{\varepsilon})$ (rather than $O(\varepsilon)$ ) where $\varepsilon=$ $K_{d} / R_{T} \ll 1$. To see this more explicitly we set $L_{T}=R_{T}$ in Eq. (8) and expand its solution as

$$
\begin{aligned}
{[L R] } & =R_{T}+\frac{K_{d}}{2}-\sqrt{K_{d} R_{T}+\frac{K_{d}^{2}}{4}} \\
& =R_{T}(1-\sqrt{\varepsilon}+O(\varepsilon)) .
\end{aligned}
$$




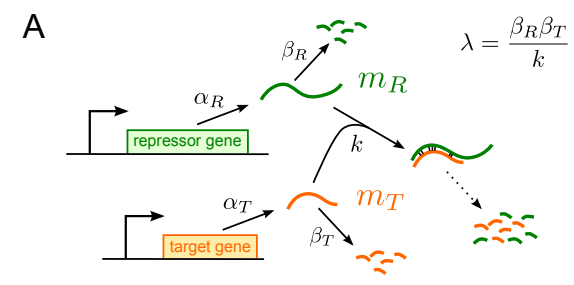

B

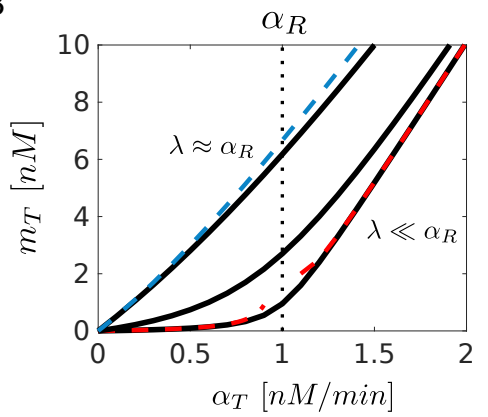

Figure 2: Tunable threshold in gene regulation. (A) Model for the repression of a target gene through complex formation between target mRNA and repressor mRNA according to (Levine et al., 2007). (B) If the target-repressor interaction is sufficiently strong $\left(\lambda \ll \alpha_{R}\right)$ a sharp threshold develops for the onset of target gene expression as a function of the synthesis rate. Solid lines were computed from Eq. (16) with the replacement $\left([L], L_{T}, R_{T}, K_{d}\right) \rightarrow\left(\beta_{T}\left[m_{T}\right], \alpha_{T}, \alpha_{R}, \lambda\right)$ for $\alpha_{R}=1 \mathrm{nM} / \mathrm{s}, \beta_{T}=0.1 / \mathrm{min}$ and $\lambda=0.01,0.1,1$ (bottom to top). Red and blue dashed lines were computed from Eqs. (17) and (18), respectively.

To reproduce this solution to leading order we modify the approximation in Eq. (10) as (cf. (Levine et al., 2007))

$$
[L R] \approx\left\{\begin{array}{ll}
L_{T}\left(1-\frac{K_{d}}{R_{T}-L_{T}+\sqrt{K_{d} R_{T}}}\right), & L_{T} \leq R_{T} \\
R_{T}\left(1-\frac{K_{d}}{L_{T}-R_{T}+\sqrt{K_{d} R_{T}}}\right), & L_{T} \geq R_{T}
\end{array} .\right.
$$

Using the same procedure one may derive improved approximations for $\tau_{s}$ (Eq. 11) and [L] (Eq. 17) as well as for many of the approximations given in the remainder of this review.

\subsection{Independent binding to a scaffold protein}

Signal transduction often involves recruitment of signaling molecules to a scaffold protein (Witzel et al., 2012) which one may think of as kinases for definiteness (Fig. 3A). If binding of each kinase to the scaffold occurs independently the probability for the occurrence of a fully assembled complex consisting of the scaffold and $n$ kinases is given by

$$
p_{n}\left(S K_{1} \ldots K_{n}\right)=\Pi_{i=1}^{n} p_{1}\left(S K_{i}\right)
$$

where $p_{1}\left(S K_{i}\right)=\left[S K_{i}\right] / S_{T}$ is the probability for binding of a single kinase to the scaffold. In that case the concentration of the fully assembled complex can be computed from (cf. (Borisov et al., 2005))

$$
\left[S K_{1} \ldots K_{n}\right]=\frac{\prod_{i=1}^{n}\left[S K_{i}\right]}{S_{T}^{n-1}}
$$

where the concentration of a single scaffold-kinase complex is determined by the LR-type equation (cf. Eq. 8)

$$
\left[S K_{i}\right]^{2}-\left[S K_{i}\right]\left(S_{T}+K_{i T}+K_{d i}\right)+S_{T} K_{i T}=0 .
$$

Here, $S_{T}$ denotes the total concentration of the scaffold while $K_{i T}$ and $K_{d i}$ denote the total concentrations of the kinases and their dissociation constants, respectively.

The approximate expressions derived for the solution of Eq. (22) can now be used to construct explicit expressions for the concentration of the fully assembled complex. To illustrate this procedure we consider the case $n=3$. If all three kinases are of high affinity (so that $K_{d i} \ll K_{i T}$ for $i=1,2,3$ ) the stimulusresponse curve for the fully assembled complex can be approximated by (cf. Fig. 3B)

$$
\left[S K_{1} K_{2} K_{3}\right] \approx\left\{\begin{array}{rr}
S_{T}, & 0<S_{T}<K_{1 T} \\
K_{1 T}, & K_{1 T}<S_{T}<K_{2 T} \\
\frac{K_{1 T} K_{2 T}}{S_{T}}, & K_{2 T}<S_{T}<K_{3 T} \\
\frac{K_{1 T} K_{2 T} K_{3 T}}{S_{T}^{2}}, & K_{3 T}<S_{T}
\end{array}\right.
$$

Here, we have assumed without loss of generality that $K_{1 T}<$ $K_{2 T}<K_{3 T}$. Interestingly, in the regime $K_{1 T}<S_{T}<K_{2 T}$ the concentration of the fully assembled complex is independent of the scaffold concentration, i.e. the system exhibits 'concentration robustness' with respect to changes in $S_{T}$ in that regime. The extent of this regime can be tuned by changing the concentrations of the two least abundant kinases (Witzel et al., 2012). Indeed, when the concentrations of all kinases become equal the two middle regimes in Eq. (23) disappear leaving a sharp maximum at $S_{T}=K_{i T}$ in the response curve (Fig. 3C). As demonstrated in Fig. 3D a plateau can also be generated with two high-affinity kinases $\left(K_{1}\right.$ and $\left.K_{2}\right)$ and one low-affinity kinase $\left(K_{3}\right)$. Combining the corresponding expressions for the high- and low-affinity approximations of Eq. (22) yields

$$
\left[S K_{1} K_{2} K_{3}\right] \approx \frac{K_{3 T}}{K_{3 T}+S_{T}+K_{d 3}}\left[S K_{1} K_{2}\right]
$$

where $\left[S K_{1} K_{2}\right]$ is given by

$$
\left[S K_{1} K_{2}\right] \approx\left\{\begin{array}{rr}
S_{T}, & 0<S_{T}<K_{1 T} \\
K_{1 T}, & K_{1 T}<S_{T}<K_{2 T} . \\
\frac{K_{1 T} K_{2 T}}{S_{T}}, & K_{2 T}<S_{T}
\end{array} .\right.
$$

However, since $K_{3 T} \ll K_{d 3}$ for the low-affinity kinase the activity of the fully assembled complex in the plateau regime is substantially reduced by a factor $K_{3 T} / K_{d 3} \ll 1$ compared to the case of three high-affinity kinases (Eq. 23).

\subsection{Cooperativity and molecular exchange systems}

If binding of one ligand affects binding of another ligand the system is said to exhibit cooperativity. The analysis of such a scenario is more complicated and even in the simplest case of two ligands (Fig. 4A) no general solution exists. Depending on whether binding of the first ligand favors or hinders binding of the next one the cooperativity can be positive or negative, respectively. The cooperative-binding motif arises in many different contexts. Classically, it described mixed-type inhibition of an enzyme-catalyzed reaction (Cornish-Bowden, 2004) 
A

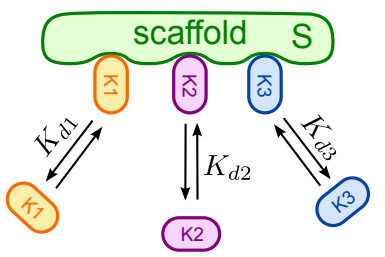

B

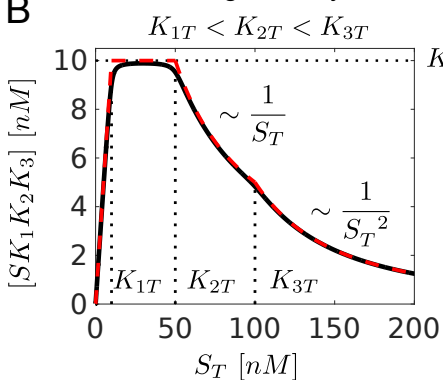

C $3 \times$ high-affinity

$K_{1 T}=K_{2 T}=K_{3 T}$

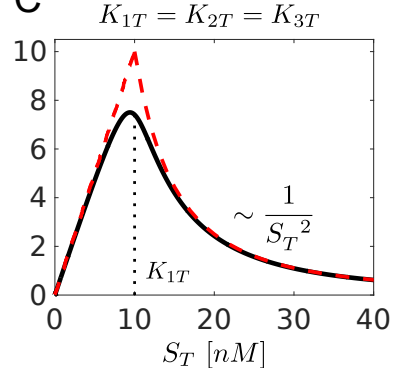

$2 \times$ high- $/ 1 \times$ low-affinity

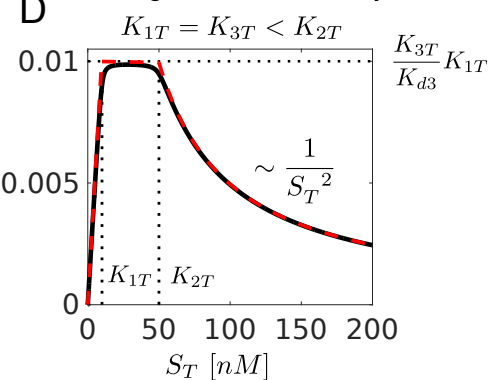

Figure 3: Ligand binding to a scaffold protein may lead to concentration robustness. (A) Three kinases (K1-K3) bind independently to a scaffold S with dissociation constants $K_{d i}$. (B-D) Stimulus-response curves for the fully assembled complex as a function of the scaffold concentration. If at least two high-affinity kinases bind to the scaffold a plateau regime exists in the region $K_{1 T}<S_{T}<K_{2 T}$ where the concentration of the fully assembled complex does not depend on the scaffold concentration. Parameters: (B) $K_{1 T}=10 n M, K_{2 T}=50 n M, K_{3 T}=100 n M, K_{d i}=0.1 n M$. (C) $K_{i T}=10 n M, K_{d i}=0.1 n M$. (D) $K_{1 T}=K_{3 T}=10 n M, K_{2 T}=50 n M$, $K_{d 1}=K_{d 2}=0.1 n M, K_{d 3}=10^{4} n M$.

A

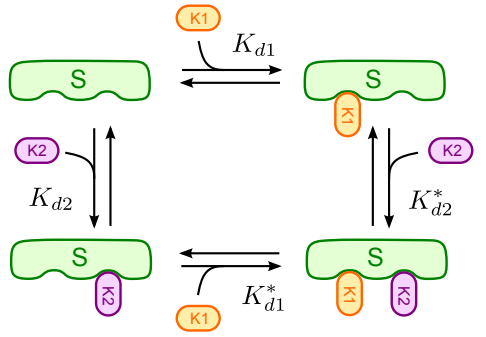

B

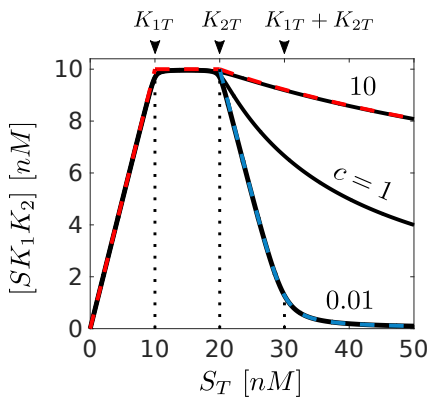

C

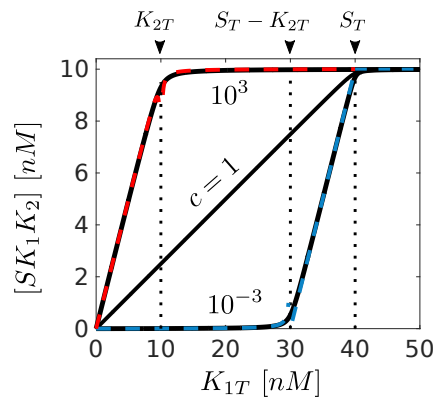

Figure 4: Cooperativity between ligands binding to a scaffold. (A) Two kinases bind to a scaffold such that $c=K_{d 2} / K_{d 1}^{*}>1$ (positive cooperativity) or $c<1$ (negative cooperativity). (B) Negative cooperativity leads to more localized concentration profiles for $\left[S K_{1} K_{2}\right]$ as a function of the scaffold concentration $\left(K_{1 T}=10 n M\right.$, $\left.K_{2 T}=20 n M\right)$. (C) Strong negative cooperativity generates a sharp threshold and ultrasensitivity for $\left[S K_{1} K_{2}\right]$ as a function of the kinase concentration $\left(S_{T}=40 n M\right.$, $\left.K_{2 T}=10 n M\right)$. Note that stimulus-response curves are partially overlapping for $S_{T} \leq K_{2 T}(\mathrm{~B})$ and $K_{1 T} \geq S_{T}(\mathrm{C})$. Dashed lines were computed according to Eqs. (28) (B) as well as Eq. (31) (C, red curve) and Eq. (32) (C, blue curve). Other parameters: $b=1, K_{d 1}^{*}=0.01 n M$. 
where $S, K_{1}$ and $K_{2}$ correspond to the enzyme, the substrate and the inhibitor, respectively. The same pattern was also used in a pharmacological context to estimate the binding affinities of drugs that interact allosterically with a receptor (Ehlert, 1988). Recently, this motif has been studied in the context of twocomponent signaling where a signaling molecule controls the interaction between a modulator and a response regulator $(\mathrm{Ba}-$ bel and Bischofs, 2016).

The steady states of the cooperative-binding motif are described by 3 of the 4 binding equilibria, e.g.

$$
\begin{aligned}
{[S]\left[K_{1}\right] } & =K_{d 1}\left[S K_{1}\right] \\
{\left[S K_{1}\right]\left[K_{2}\right] } & =K_{d 2}^{*}\left[S K_{1} K_{2}\right] \\
{\left[S K_{2}\right]\left[K_{1}\right] } & =K_{d 1}^{*}\left[S K_{1} K_{2}\right]
\end{aligned}
$$

together with the three conservation relations

$$
\begin{aligned}
{[S]+\left[S K_{1}\right]+\left[S K_{2}\right]+\left[S K_{1} K_{2}\right] } & =S_{T} \\
{\left[K_{1}\right]+\left[S K_{1}\right]+\left[S K_{1} K_{2}\right] } & =K_{1 T} \\
{\left[K_{2}\right]+\left[S K_{2}\right]+\left[S K_{1} K_{2}\right] } & =K_{2 T} .
\end{aligned}
$$

Since the free energy change for the formation of the ternary complex $\left(S K_{1} K_{2}\right)$ must not depend on the order in which it was formed the 4 th dissociation constant $K_{d 2}$ is determined by the other three constants through the detailed balance relation

$$
K_{d 1} \cdot K_{d 2}^{*}=K_{d 2} \cdot K_{d 1}^{*} \text {. }
$$

To characterize the steady state operating regimes of the cooperative-binding motif it is useful to introduce two parameters that measure cooperativity $(c)$ and bias $(b)$ as

$$
c=\frac{K_{d 2}}{K_{d 1}^{*}}, \quad b=\frac{K_{d 2}}{K_{d 1}}=\frac{K_{d 2}^{*}}{K_{d 1}^{*}} .
$$

Similar as in the case of the receptor-ligand binding motif one may distinguish a high-affinity and a low-affinity regime (cf. Fig. 1). Together with the limiting regimes of strong positive $(c \gg 1)$ or strong negative $(c \ll 1)$ cooperativity and strong bias $(b \ll 1)$ or no bias $(b=1)$ there are potentially 8 interesting operating regimes for this motif.

Recently, Ha et al. showed that in the high-affinity regime $\left(K_{d 1}^{*} \ll S_{T}\right)$ the steady states of the ternary complex [S $\left.K_{1} K_{2}\right]$, defined by Eqs. (25) and (26), can be approximated by the set $\mathcal{S}=\left\{S_{T}, K_{1 T}, K_{2 T}, x_{+}, x_{-}\right\}$where $x_{ \pm}$are the roots of the quadratic equation (Ha et al., 2016)

$$
x^{2}-\left(K_{1 T}+K_{2 T}+\frac{b}{c-b} S_{T}\right) x+\frac{c}{c-b} K_{1 T} K_{2 T} \approx 0 .
$$

This equation can be viewed as a generalized LR-type equation where the constant term involves the additional scaling factor $c /(c-b)$ (cf. Eq. 1). The elements in $\mathcal{S}$ can be combined to yield approximate expressions for the stimulus-response curve as a function of various parameters. For example, if $K_{1 T}<K_{2 T}$ the concentration of $S K_{1} K_{2}$ as a function of $S_{T}$ is given by

$$
\left[S K_{1} K_{2}\right] \approx\left\{\begin{array}{cr}
S_{T}, & 0<S_{T}<K_{1 T} \\
K_{1 T}, & K_{1 T}<S_{T}<K_{2 T} \\
x_{ \pm}, & K_{2 T}<S_{T}
\end{array}\right.
$$

where $x_{ \pm}$denote the positive $\left(x_{+}\right)$or negative $\left(x_{-}\right)$square root solution of Eq. (27) depending on whether $c<b$ or $c>b$, respectively (Fig. 4B). If $c=b$ Eq. (27) has just the single solution $x=K_{1 T} K_{2 T} / S_{T}$ which declines to zero as $1 / S_{T}$, similarly as in the case of independent binding (cf. Eq. 24). In the case of strong positive cooperativity $(c \gg b)$ the asymptotic decline of $\left[S K_{1} K_{2}\right]$ is described by (cf. subsection 6.2)

$$
x_{-} \sim \frac{K_{1 T} K_{2 T}}{K_{1 T}+K_{2 T}+\varepsilon S_{T}}, \quad S_{T} \gg \frac{K_{1 T}+K_{2 T}}{\varepsilon}
$$

where $\varepsilon \equiv b /(c-b) \ll 1$, i.e. the concentration of the ternary complex decays more slowly as in the case of independent binding (Fig. 4B). In contrast, for strong negative cooperativity $(c \ll b)$ the solution of Eq. (27) can be approximated by

$$
x_{+} \approx\left\{\begin{array}{cr}
K_{1 T}+K_{2 T}-S_{T}, & K_{2 T} \leq S_{T}<K_{1 T}+K_{2 T} \\
\eta \frac{K_{1 T} K_{2 T}}{S_{T}-\left(K_{1 T}+K_{2 T}\right)}, & S_{T}>K_{1 T}+K_{2 T}
\end{array}\right.
$$

where $\eta \equiv c /(b-c) \ll 1$, i.e. in the regime $K_{2 T} \leq S_{T}<K_{1 T}+$ $K_{2 T}$ the concentration of the ternary complex decreases almost linearly leading to a more "localized" concentration profile.

Alternatively, when considered as a function of the kinase concentration $K_{1 T}$ under conditions when the scaffold is not saturated $\left(S_{T}>K_{2 T}\right)$ strong positive cooperativity generates a stimulus-response curve

$$
\left[S K_{1} K_{2}\right] \approx\left\{\begin{array}{cc}
K_{1 T}\left(1-\varepsilon \frac{S_{T}-K_{2 T}}{K_{2 T}-K_{1 T}}\right), & 0 \leq K_{1 T}<K_{2 T} \\
K_{2 T}\left(1-\varepsilon \frac{S_{T}-K_{1 T}}{K_{1 T}-K_{2 T}}\right), & K_{2 T} \leq K_{1 T}<S_{T} \\
K_{2 T}, & S_{T} \leq K_{1 T}
\end{array}\right.
$$

which is of linear-saturation shape (Fig. 4C, red curve) similar as obtained for receptor-ligand binding in the case of a highaffinity ligand (Fig. 1B). In the limit of strong negative cooperativity the approximation for the stimulus-response curve reads

$$
\left[S K_{1} K_{2}\right] \approx\left\{\begin{array}{cc}
\eta \frac{K_{1 T} K_{2 T}}{\left(S_{T}-K_{2 T}\right)-K_{1 T}}, & 0 \leq K_{1 T}<S_{T}-K_{2 T} \\
K_{1 T}-\left(S_{T}-K_{2 T}\right), & S_{T}-K_{2 T}<K_{1 T}<S_{T} \\
K_{2 T}, & S_{T} \leq K_{1 T}
\end{array}\right.
$$

which shows that in the transition region, defined by $S_{T}-K_{2 T}<$ $K_{1 T}<S_{T}$, the response curve changes in an ultrasensitive manner from $O(\eta)$ to $K_{2 T}$ (Fig. 4C, blue curve). The width of the transition region as well as the maximal amplitude of the response are given by $K_{2 T}$, i.e. steep responses require $K_{2 T} \ll S_{T}$ which limits the dynamic range of the output. By combining theory with experiments $\mathrm{Ha}$ and Ferrell recently showed that negative cooperativity may lead to sharp thresholds and ultrasensitivity in the cooperative binding motif for the case of two identical ligands (Ha and Ferrell Jr., 2016).

Another class of systems that operates under conditions of strong negative cooperativity are molecular exchange systems (Fig. 5). A prominent example are small GTPases which alter between an inactive GDP-bound form and a GTP-bound form that can act as a signaling molecule for a large variety of cellular processes (Cherfils and Zeghouf, 2013). In isolation the binary GTPase.GDP / GTPase.GTP complexes are extremely stable with half-lifes between hours and days (Goody 


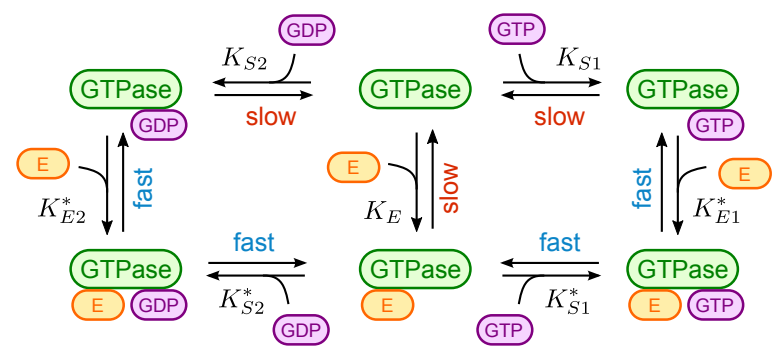

Figure 5: Molecular exchange system operate under conditions of strong negative cooperativity $\left(K_{S i}^{*} \gg K_{S i}, \mathrm{i}=1,2\right)$. Binding of the exchange factor $\mathrm{E}$ to the GTPase lowers the binding affinities for GTP and GDP from the ternary complexes. In the presence of a high GTP/GDP ratio E mediates the exchange of GDP for GTP favoring the formation of the GTP.GTPase state.

and Hofmann-Goody, 2002). However, in the presence of a guanine nucleotide exchange factor (GEF) the dissociation constants of GDP and GTP from the GTPase are increased by several orders of magnitude leading to their rapid dissociation and to nucleotide exchange (Fig. 5). Mathematical models have shown that there exist intrinsic trade-offs in molecular exchange systems (Goryachev and Pokhilko, 2006; Straube et al., 2017) which, in the case of the GTPase cycle, predict an optimal concentration for the GEF as a result of a trade-off between high GTPase activity and fast GDP/GTP cycling.

\subsection{Substrate competition and thresholds}

Substrate competition arises if at least two ligands compete for a single binding site on a receptor (Fig. 6A). Examples of substrate competition are numerous including gene regulation by small RNAs (Levine et al., 2007; Mukherji et al., 2011) and during development (Kim et al., 2010, 2011), binding of substrate receptors to ubiquitin E3 ligases (Petroski and Deshaies, 2005; Lydeard et al., 2013), phosphorylation/dephosphorylation cycles (Kim and Ferrell, Jr., 2007; Rowland et al., 2012) and bacterial two-component systems (Laub and Goulian, 2007; Rowland and Deeds, 2014), the latter two being discussed in sections 3.3 and 4.3, respectively.

Here, we consider the simplest form of substrate competition as it arises when two ligands bind to a scaffold protein or to a receptor (Fig. 6A). In that case the steady states are determined by the two binding equilibria

$$
K_{d 1}[L 1 . R]=[L 1][R], \quad K_{d 2}[L 2 . R]=[L 2][R]
$$

and the three conservation relations

$$
\begin{aligned}
{[L 1]+[L 1 . R] } & =L 1_{T} \\
{[L 2]+[L 2 . R] } & =L 2_{T} \\
{[R]+[L 1 . R]+[L 2 . R] } & =R_{T} .
\end{aligned}
$$

Combining Eqs. (33) and (34) yields a cubic polynomial which can be written in the form (Straube, 2015)

$$
(1-\varepsilon)[L 1 . R]^{3}-a_{2}[L 1 . R]^{2}-a_{1}[L 1 . R]+\varepsilon R_{T} L 1_{T}^{2}=0
$$

where $a_{2}$ and $a_{1}$ are given by

$$
\begin{aligned}
& a_{2}=-\left(L 1_{T}+R_{T}-L 2_{T}+K_{d 1}\right)+\varepsilon\left(2 L 1_{T}+R_{T}+K_{d 1}\right) \\
& a_{1}=L 1_{T}\left(R_{T}-L 2_{T}\right)-\varepsilon L 1_{T}\left(L 1_{T}+2 R_{T}+K_{d 1}\right) .
\end{aligned}
$$

Depending on the value of $\varepsilon=K_{d 2} / K_{d 1}$ some interesting limiting regimes can be considered.

\subsubsection{Competition by relative abundance}

If both ligands exhibit the same affinity $\left(K_{d 1}=K_{d 2} \equiv K_{d}\right)$, so that competition only arises through relative ligand abundances, the cubic polynomial reduces to a $\mathbf{L R}$-type equation

$$
[L 1 . R]^{2}-[L 1 . R]\left(L 1_{T}+\rho R_{T}+\rho K_{d}\right)+\rho R_{T} L 1_{T}=0,
$$

i.e. compared to Eq. (8) the receptor concentration $R_{T}$ and the dissociation constant $K_{d}$ are rescaled by a factor

$$
\rho=\frac{L 1_{T}}{L 1_{T}+L 2_{T}}
$$

which measures the relative abundance of L1. From the expressions in Eqs. (10) and (12) one may readily obtain the corresponding approximations in the high-affinity $\left(K_{d} \ll R_{T}\right)$ and low-affinity regimes $\left(K_{d} \gg R_{T}\right)$ as

$$
[L 1 . R] \approx\left\{\begin{array}{cl}
L 1_{T}, & L 1_{T}<R_{T}-L 2_{T} \\
\frac{L 1_{T}}{L 1_{T}+L 2_{T}} R_{T}, & L 1_{T}>R_{T}-L 2_{T}
\end{array}\right.
$$

and

$$
[L 1 . R] \approx \frac{\left(L 1_{T}+L 2_{T}\right) R_{T}}{L 1_{T}+L 2_{T}+R_{T}+K_{d}}
$$

respectively. Hence, under conditions when the receptor is saturated $\left(L 1_{T}+L 2_{T}>R_{T}\right)$ the receptor-ligand complex for highaffinity ligands (Eq. 36) increases proportional to the relative abundance of a ligand - in agreement with intuition.

\subsubsection{Strong substrate competition}

If the affinity of one ligand is much higher than that of the other one the solutions of the cubic equation (Eq. 35) can be analyzed in the limit of strong substrate competition $\varepsilon \ll 1$ which may lead to thresholds and ultrasensitivity (Straube, 2015). For receptor concentrations below the threshold (which occurs at $R_{T}=L 2_{T}$ ) the concentration of $L 1 . R$ remains low

$$
[L 1 . R] \approx \varepsilon \frac{L 1_{T} R_{T}}{L 2_{T}-R_{T}}, \quad R_{T}<L 2_{T}
$$

since $L 2$ sequesters all receptors away. Beyond the treshold $\left(R_{T}>L 2_{T}\right)$ the response curve can, again, be approximated by the solution of a LR-type equation

$$
[L 1 . R]^{2}-[L 1 . R]\left(L 1_{T}+R_{T}^{e f f}+K_{d 1}\right)+L 1_{T} R_{T}^{e f f} \approx 0
$$

where $R_{T}^{\text {eff }}=R_{T}-L 2_{T}$ denotes the effective receptor concentration. The latter acounts for the fact that the low-affinity ligand can only bind to receptor molecules that are not yet bound by high-affinity ligands.

Depending on the relative magnitude between $K_{d 1}$ and $L 1_{T}$ one may use similar approximations for the solutions of Eq. (38) as for the single ligand in Eqs. (10) and (12). Hence, if $K_{d 1} \gg L 1_{T}$ the response curve increases hyperbolically beyond the threshold (Fig. 6B) while for $K_{d 1} \ll L 1_{T}$ the response curve 
may become highly ultrasensitive (Fig. 6C). In the latter regime one obtains the approximation

$$
[L 1 . R] \approx\left\{\begin{array}{rr}
\varepsilon \frac{L 1_{T} R_{T}}{L 2_{T}-R_{T}}, & 0 \leq R_{T}<L 2_{T} \\
R_{T}-L 2_{T}, & L 2_{T}<R_{T}<L 1_{T}+L 2_{T} \\
L 1_{T}, & L 1_{T}+L 2_{T} \leq R_{T} .
\end{array}\right.
$$

Comparing this stimulus-response curve with that for negative cooperativity (Eq. 32) we see that both exhibit a tripartite structure with a linear increase in the middle regime. In particular, there exists a similar trade-off between the steepness of the response curve, which decreases with $L 1_{T}$, and the dynamic range, which increases with $L 1_{T}$.

The response curve for $[L 1 . R]$ as a function of the ligand concentration $L 1_{T}$ can exhibit biphasic behavior and is, thus, more difficult to obtain (cf. section 6.3). Note that the asymptotic value for $[L 1 . R]$ in the limit of $L 1_{T} \gg K_{d 1}$, as predicted by Eq. (38), is $R_{T}^{\text {eff }}$ rather than $R_{T}$. Hence, the latter describes the relation between $[L 1 . R]$ and $L 1_{T}$ only at sufficiently low ligand concentrations. A more detailed analysis of the cubic equation (Eq. 35) shows that the behavior of [L1.R] for large $L 1_{T}$ can be approximated by the solution of the $\mathbf{L}$-type equation (Fig. 6D) (cf. subsection 6.3)

$$
[L 1 . R]^{2}+[L 1 . R]\left(L 2_{T}+\varepsilon L 1_{T}-R_{T}\right)-\varepsilon L 1_{T} R_{T}=0 .
$$

When $R_{T}<L 2_{T}$ one may obtain an approximate solution of this equation by balancing the linear and the constant term

$$
[L 1 . R] \approx \frac{L 1_{T} R_{T}}{L 1_{T}+\frac{L 2_{T}-R_{T}}{\varepsilon}}, \quad R_{T}<L 2_{T} .
$$

In that case the approximation is valid for all ligand concentrations and the stimulus-response curve exhibits no biphasic behavior (Fig. 6E).

\section{Covalent Modification Cycles}

\subsection{Goldbeter-Koshland model and the GK-type equation}

In 1981 Goldbeter and Koshland proposed a simple mechanism through which biological systems can generate extremely high sensitivity (known as zero-order ultrasensitivity) to input signals (Goldbeter and Koshland Jr., 1981). Interestingly, the mechanism did not involve cooperative binding of ligands to an oligomeric enzyme rather than two (converter) enzymes catalyzing the covalent modification and demodification of a target protein by utilizing energy in the form of ATP. Often, such futile cycles occur in the form of phosphorylation / dephosphorylation cycles as mediated by a kinase and a phosphatase, respectively (Fig. 7), but energy in the form of ATP is also required for other covalent modifications such as ubiquitylation or neddylation (Dye and Schulman, 2007).

The dynamics of phosphorylation/dephosphorylation cycles,

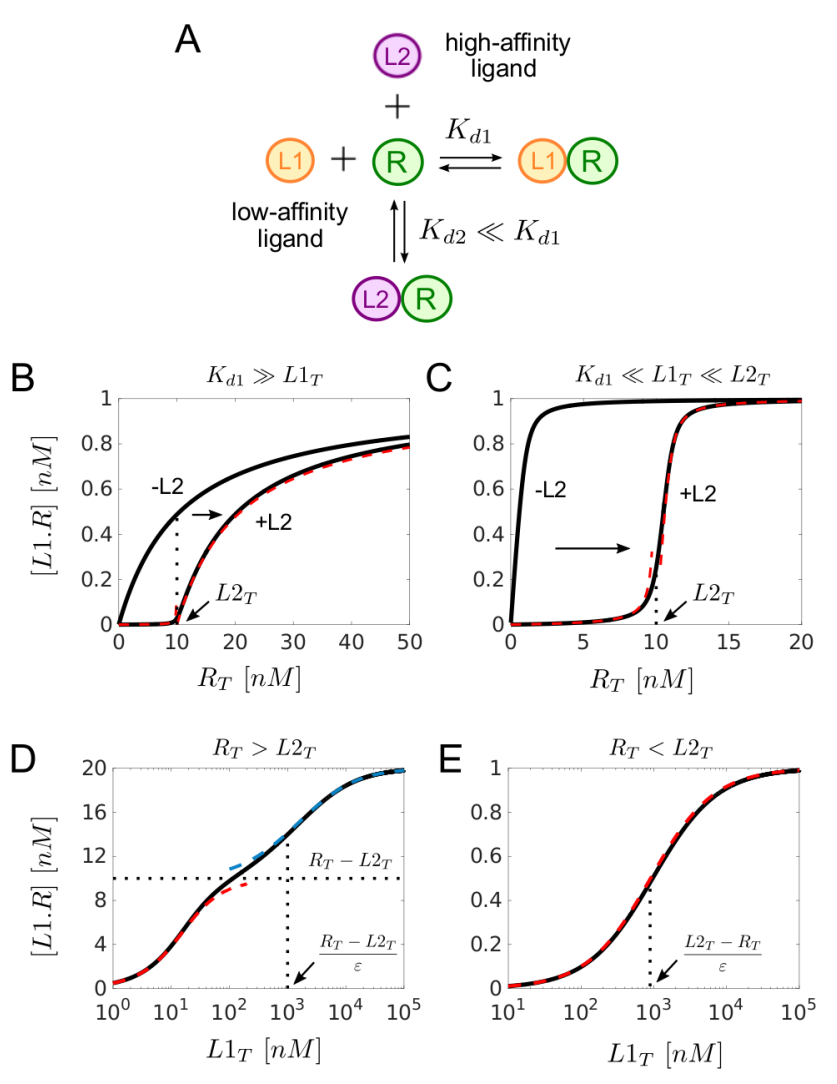

Figure 6: Generation of a threshold and ultrasensitivity through substrate competition. (A) Two ligands (L1 and L2) compete for binding to a receptor (R). (B,C) Steady state concentration of the ligand-receptor complex as a function of receptor concentration for $K_{d 1}=10 n M, K_{d 2}=0.01 n M(\mathrm{~B})$ and $K_{d 1}=0.1 \mathrm{nM}$, $K_{d 2}=0.001 n M(\mathrm{C})$ so that $\varepsilon=K_{d 2} / K_{d 1} \ll 1$. \pm L2 indicate the presence (+) or absence $(-)$ of $L 2\left(L 2_{T}=0\right)$. Other parameters: $L 1_{T}=1 n M, L 2_{T}=10 n M$. Red dashed lines were drawn according to Eqs. (37) and (38). (D,E) $[L 1 . R]$ as a function of the concentration of the low-affinity ligand for $R_{T}=20 \mathrm{nM}$ (D) and $R_{T}=1 n M(\mathrm{E})$. Other parameters: $L 2_{T}=10 n M, K_{d 1}=10 n M, K_{d 2}=0.1 n M$. Blue dashed curve was drawn from Eq. (40) while red dashed lines correspond to Eqs. (38) (D) and (41) (E).
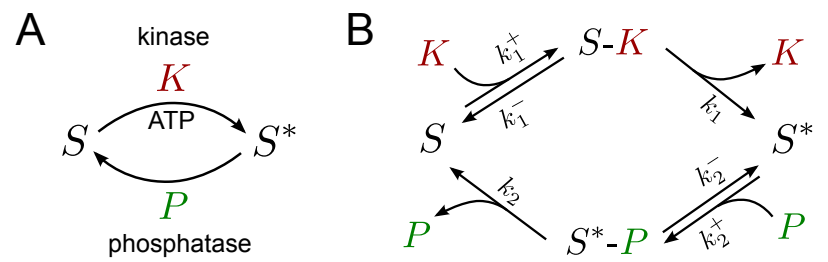

Figure 7: Covalent modification cycle: (A) The substrate $S$ is phosphorylated by a kinase $(\mathrm{K})$ with ATP as a cofactor and dephosphorylated by a phosphatase (P). (B) Detailed reaction scheme assuming both kinase and phosphatase reaction follow a Michaelis-Menten scheme and the ATP concentration is constant. 
as described by Fig. 7B, is determined by the ODE system

$$
\begin{aligned}
\frac{d\left[S^{*}\right]}{d t} & =k_{1}[S K]-k_{2}^{+}\left[S^{*}\right][P]+k_{2}^{-}\left[S^{*} P\right] \\
\frac{d[S K]}{d t} & =k_{1}^{+}[S][K]-\left(k_{1}^{-}+k_{1}\right)[S K] \\
\frac{d\left[S^{*} P\right]}{d t} & =k_{2}^{+}\left[S^{*}\right][P]-\left(k_{2}^{-}+k_{2}\right)\left[S^{*} P\right]
\end{aligned}
$$

where $[S],[K]$ and $[P]$ have to be replaced using the conservation relations

$$
\begin{aligned}
{[S]+\left[S^{*}\right]+[S K]+\left[S^{*} P\right] } & =S_{T} \\
{[K]+[S K] } & =K_{T} \\
{[P]+\left[S^{*} P\right] } & =P_{T} .
\end{aligned}
$$

The Goldbeter-Koshland model is based on two assumptions: (i) the enzyme-substrate complexes rapidly approach a quasistationary state defined by

$$
\frac{d[S K]}{d t} \approx \frac{d\left[S^{*} P\right]}{d t} \approx 0,
$$

and the substrate concentration is much higher than that of the converter enzymes, i.e.

$$
S_{T} \gg \max \left(K_{T}, P_{T}\right),
$$

which allows simplifying the conservation relation for the substrate as $[S]+\left[S^{*}\right] \approx S_{T}$. Under these conditions Goldbeter and Koshland derived the following ODE for the concentration of $S^{*}$ (Goldbeter and Koshland Jr., 1981)

$$
\frac{d\left[S^{*}\right]}{d t} \approx k_{1} K_{T} \frac{S_{T}-\left[S^{*}\right]}{K_{1}+S_{T}-\left[S^{*}\right]}-k_{2} P_{T} \frac{\left[S^{*}\right]}{K_{2}+\left[S^{*}\right]} .
$$

Here, $K_{T}, P_{T}$ and $S_{T}$ denote the total concentrations of kinase, phosphatase and substrate while $K_{i}=\left(k_{i}+k_{i}^{-}\right) / k_{i}^{+}$are the corresponding Michaelis-Menten constants. The steady state equation $\left(d\left[S^{*}\right] / d t=0\right)$ derived from Eq. (44) will be denoted as GK-type equation. Note that it is structurally similar to Eq. (3) mentioned in the Introduction.

To characterize the steady state operating regimes of covalent modification cycles it is useful to introduce dimensionless quantities through $x=\left[S^{*}\right] / S_{T}$ and $K_{i}^{*}=K_{i} / S_{T}$ which yields the steady state equation

$$
x^{2}-\left(1+\frac{K_{1}^{*}+\alpha K_{2}^{*}}{1-\alpha}\right) x+\frac{\alpha K_{2}^{*}}{1-\alpha}=0, \quad \alpha=\frac{k_{1} K_{T}}{k_{2} P_{T}}
$$

where $\alpha$ is the ratio between the maximal rates of kinase and phosphatase. The solution of Eq. (45) reads

$$
x= \begin{cases}x_{-}, & \alpha<1 \\ x_{+}, & \alpha>1\end{cases}
$$

where $x_{ \pm}$are given by

$$
x_{ \pm}=\frac{1}{2}\left(1+\frac{K_{1}^{*}+\alpha K_{2}^{*}}{1-\alpha}\right) \pm \frac{1}{2} \sqrt{\left(1+\frac{K_{1}^{*}+\alpha K_{2}^{*}}{1-\alpha}\right)^{2}-4 \frac{\alpha K_{2}^{*}}{1-\alpha}} .
$$

A systematic analysis of the steady state operating regimes of covalent modification cycles was given by Gomez-Uribe et al. (Gomez-Uribe et al., 2007). Depending on whether the converter enzymes are saturated $\left(K_{i}^{*} \ll 1\right)$ or unsaturated $\left(K_{i}^{*} \gg 1\right)$ there exist 4 operating regimes as depicted in Fig. (8). Zeroorder ultrasensitivity requires both enzymes to be saturated $\left(\max \left(K_{1}^{*}, K_{2}^{*}\right) \ll 1\right)$. On the other hand if both enzymes are unsaturated $\left(\min \left(K_{1}^{*}, K_{2}^{*}\right) \gg 1\right)$ the response curve resembles that of a Michaelian enzyme (Fig. 8B).

Interestingly, if the two converter enzymes operate in opposite regimes the steady state equation resulting from Eq. (44) becomes similar to one of the two quadratic equations shown in Eqs. (1) and (2). Specifically, if the phosphatase is saturated while the kinase is unsaturated $\left(K_{2}^{*} \ll 1 \ll K_{1}^{*}\right)$ linearization of the first term in Eq. (44) results in the $\mathbf{L}$-type equation

$$
x^{2}+\left(\frac{K_{1}^{*}}{\alpha}+K_{2}^{*}-1\right) x-K_{2}^{*} \approx 0 .
$$

Since, by assumption, $K_{2}^{*} \ll 1$ the response curve exhibits a threshold defined by $K_{1}^{*} / \alpha=1$ beyond which the response curve increases hyperbolically as a function of $\alpha$ (Fig. 8C). In the opposite case $\left(K_{1}^{*} \ll 1 \ll K_{2}^{*}\right)$ linearization of the second term in Eq. (44) results in the LR-type equation

$$
x^{2}-\left(\alpha K_{2}^{*}+K_{1}^{*}+1\right) x+\alpha K_{2}^{*} \approx 0 .
$$

Since, by assumption, $K_{1}^{*} \ll 1$ the response curve is of linearsaturation type (Fig. 8D) similar to that of the receptor-ligand complex in the limit of a high-affinity ligand (Fig. 1B). In this case the saturation threshold is defined by $\alpha K_{2}^{*}=1$.

\subsection{High enzyme concentrations}

One of the limitations of the Goldbeter-Koshland model is that it only applies if the substrate concentration is much higher than that of the converter enzymes. While this condition is routinely used for in vitro experiments it is rarely satisfied in vivo (Blüthgen et al., 2006; Legewie et al., 2008). To analyze enzyme-catalyzed reactions at high enzyme concentrations Borghans et al. proposed a simple method, known as the total quasi-steady state approximation (tQSSA), involving a linear change of variables (Borghans et al., 1996). Their original motivation was to extend the parameter domain for the applicability of the standard QSSA (Segel and Slemrod, 1989). Later on, the conditions for the validity of the tQSSA have been refined (Schnell and Maini, 2000; Tzafriri, 2003), and the method has been successfully applied to more complicated reaction systems such as the reversible Michaelis-Menten reaction (Tzafriri and Edelman, 2004), substrate competition (Pedersen et al., 2007), covalent modification cycles (Gomez-Uribe et al., 2007; Ciliberto et al., 2007; Pedersen et al., 2010) and to describe the transient phase kinetics (Masia et al., 2016).

The tQSSA is based on the idea to introduce the total concentration of the phosphorylated substrate as a slow variable

$$
\left[Y^{*}\right]=\left[S^{*}\right]+\left[S^{*} P\right] .
$$


A
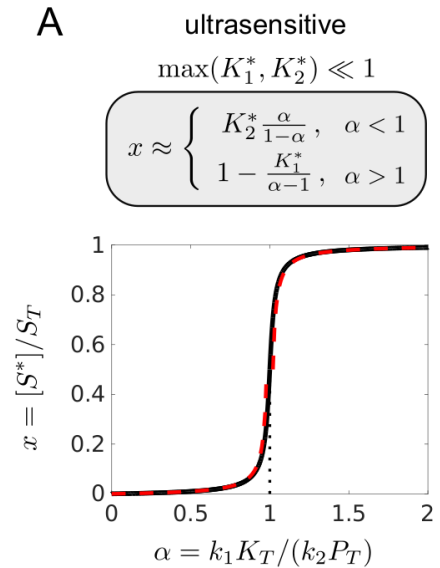

B
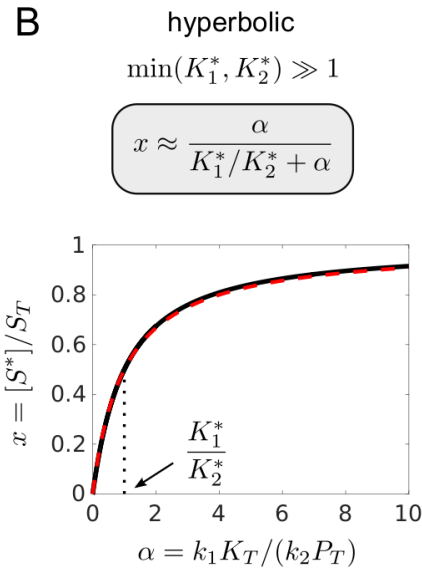

C
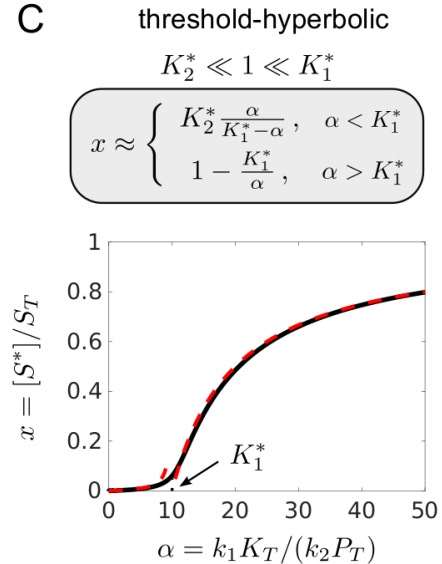

D signal-transducing
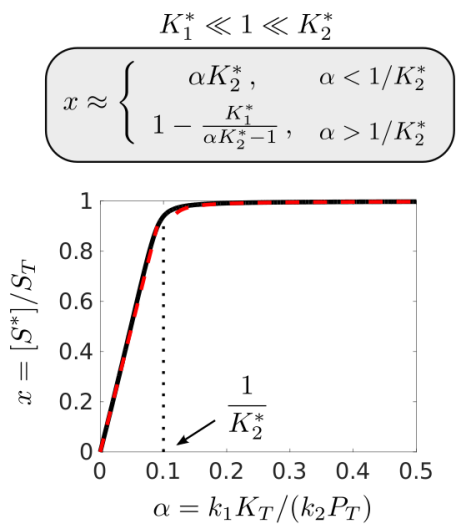

Figure 8: Steady state operating regimes of covalent modification cycles within the Goldbeter-Koshland model. Solid lines were computed from Eq. (46). Red dashed lines correspond to the approximate expressions shown above each panel. Parameters: (A) $K_{1}^{*}=K_{2}^{*}=0.01,(\mathrm{~B}) K_{1}^{*}=K_{2}^{*}=10,(\mathrm{C}) K_{1}^{*}=10, K_{2}^{*}=0.01$, (D) $K_{1}^{*}=0.01, K_{2}^{*}=10$.

Then, addition of Eqs. (42) and (43) leads to

$$
\frac{d\left[Y^{*}\right]}{d t}=k_{1}[S K]-k_{2}\left[S^{*} P\right] \text {. }
$$

Here, the concentrations of the enzyme-substrate complexes are determined by LR-type equations of the form

$$
\begin{aligned}
{[S K]^{2}-\left(K_{T}+S_{T}-\left[Y^{*}\right]+K_{1}\right)[S K] } & \\
+K_{T}\left(S_{T}-\left[Y^{*}\right]\right) & =0 \\
{\left[S^{*} P\right]^{2}-\left(P_{T}+\left[Y^{*}\right]+K_{2}\right)\left[S^{*} P\right]+P_{T}\left[Y^{*}\right] } & =0 .
\end{aligned}
$$

Substituting the positive solutions of Eqs. (50) and (51) into Eq. (49) yields the total QSSA. Compared to the standard QSSA (Eq. 44) it more accurately describes the transient dynamics of covalent modification cycles (Ciliberto et al., 2007).

However, the total QSSA can also be used to extend the classification of the steady state operating regimes given by GomezUribe et al. (Fig. 8) to the case when the concentrations of the converter enzymes are comparable to or larger than that of the substrate (Straube, 2017). To this end, we approximate the solutions of Eqs. (50) and (51) in the limit of high $\left(K_{1} \ll K_{T}\right.$ and $\left.K_{2} \ll P_{T}\right)$ and low affinity $\left(K_{1} \gg K_{T}\right.$ and $\left.K_{2} \gg P_{T}\right)$. In the first case (low- $K_{M}$ regime) this yields the approximations

$$
[S K] \approx\left\{\begin{array}{cl}
K_{T}, & {\left[Y^{*}\right]<S_{T}-K_{T}} \\
S_{T}-\left[Y^{*}\right], & {\left[Y^{*}\right]>S_{T}-K_{T}}
\end{array}\right.
$$

and

$$
\left[S^{*} P\right] \approx\left\{\begin{array}{cc}
{\left[Y^{*}\right],} & {\left[Y^{*}\right]<P_{T}} \\
P_{T}, & {\left[Y^{*}\right]>P_{T}}
\end{array} .\right.
$$

In the second case (high- $K_{M}$ regime) the approximations read

$$
\begin{aligned}
{[S K] } & \approx \frac{K_{T}\left(S_{T}-\left[Y^{*}\right]\right)}{K_{T}+S_{T}-\left[Y^{*}\right]+K_{1}} \\
{\left[S^{*} P\right] } & \approx \frac{P_{T}\left[Y^{*}\right]}{P_{T}+\left[Y^{*}\right]+K_{2}} .
\end{aligned}
$$

Altogether this defines 4 operating regimes depending on the ratio between the Michaelis-Menten constants and the concentrations of the converter enzymes. To derive approximate expressions for $\left[Y^{*}\right]$ in a particular regime one has to combine the expressions in Eqs. (52) - (55) in an appropriate manner. Depending on the parameter of interest and the values of the remaining parameters several subregimes may exist. For example, if both enzymes are saturated $\left(K_{1} \ll K_{T}\right.$ and $\left.K_{2} \ll P_{T}\right)$ and the total enzyme concentration exceeds that of the substrate $\left(K_{T}+P_{T}>S_{T}\right)$ there are 4 subregimes defining $\left[Y^{*}\right]$ as a function of $k_{1}$ depending on whether $K_{T} \lessgtr S_{T}$ and $P_{T} \lessgtr S_{T}$ (Fig. 9). Interestingly, when at least one of the enzyme concentrations is lower than that of the substrate the response curve exhibits enhanced sensitivity with $n_{H}>1$ (Fig. 9A-C). Conversely, when at least one of the enzyme concentrations is larger than that of the substrate the response curve is independent of that enzyme concentration, i.e. the system exhibits concentration robustness with respect to changes in that particular enzyme concentration. For example, if $K_{T}>S_{T}$ the response curve becomes independent of $K_{T}$ although it still depends on $P_{T}$ (Fig. 9B). Finally, if both enzyme concentrations exceed that of the substrate the response curve only depends on the catalytic rate constants and the substrate concentration (Fig. 9D). Together, this suggests that there are two regimes where covalent modification cycles exhibits both enhanced sensitivity and concentration robustness (Fig. 9B and C).

To describe the effect of genetic perturbations one is often interested in $\left[Y^{*}\right]$ as a function of the total substrate concentration $S_{T}$. One can show that in this case the shape of the response curve depends on the ratio $\alpha$ of the maximal reaction rates of kinase and phosphatase (Eq. 45). If $\alpha>1(\alpha<1)$ the response curve is monotonically increasing (decreasing) and if both converter enzymes operate in opposite regimes it may also change in a non-monotonic manner (Straube, 2017). 
A
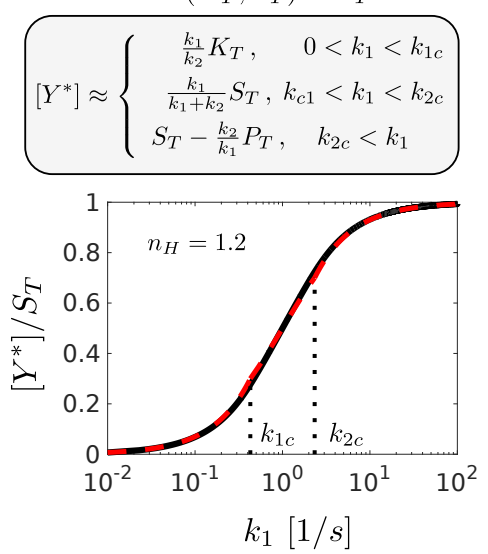

B
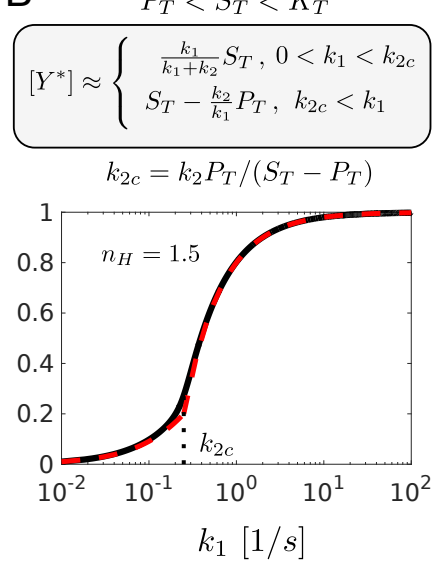

C
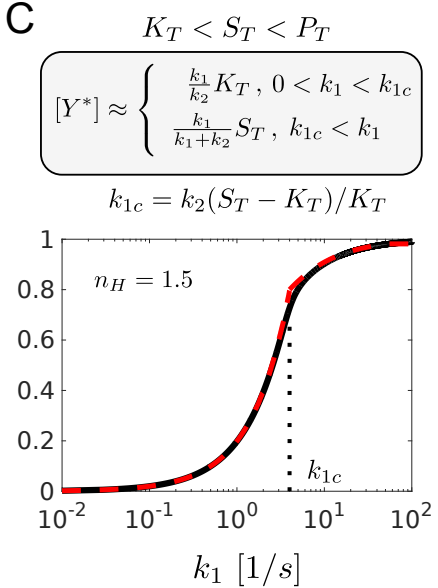

D
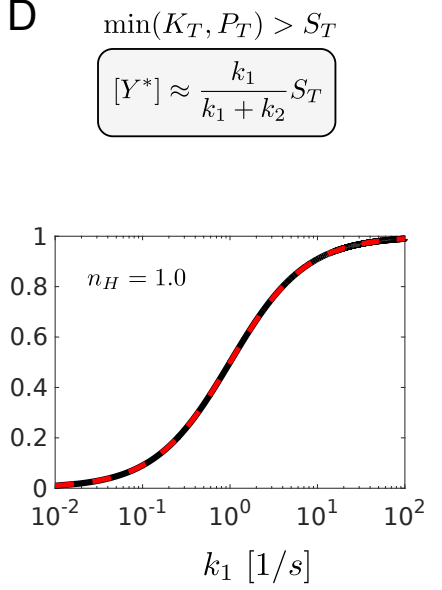

Figure 9: Steady state operating regimes of covalent modification cycles under the conditions of enzyme excess $\left(K_{T}+P_{T}>S_{T}\right)$ and enzyme saturation $\left(K_{1} \ll K_{T}\right.$ and $\left.K_{2} \ll P_{T}\right)$. The Hill coefficient $n_{H}=\ln 81 / \ln R_{S}$ is defined in terms of the response coefficient $R_{S}=k_{1}^{(90)} / k_{1}^{(10)}$ which is given by the ratio of parameter values that elicit $90 \%$ (and 10\%) of the maximal response. Red dashed lines correspond to the approximate solutions shown above each panel. Parameters: (A) $K_{T}=P_{T}=0.7 \mu M$, (B) $K_{T}=2 \mu M, P_{T}=0.2 \mu M(\mathrm{C}) K_{T}=0.2 \mu M, P_{T}=2 \mu M$ (D) $K_{T}=P_{T}=2 \mu M$. Other parameters: $S_{T}=1 \mu M, K_{1}=K_{2}=0.01 \mu M, k_{2}=1 / s$.

\subsection{Substrate competition}

Apart from the zero-order effect substrate competition represents an alternative mechanism for the generation of ultrasensitivity in regulatory networks. First experimental evidence for this effect came from studies of Kim and Ferrell who showed that the phosphorylation level of the mitotic regulator Wee1 exhibits a sharp threshold and ultrasensitivity to changes in the concentration of Cdk1 (Kim and Ferrell, Jr., 2007). The latter is a cell-cycle related kinase that is estimated to have about 200 substrates in budding yeast (Ubersax et al., 2003; Enserink and Kolodner, 2010). Interestingly, the ultrasensitive response could be reproduced in a reconstituted system by adding a different high-affinity Cdk1 substrate. However, in the absence of such substrates the response of phosphorylated Wee1 was hyperbolic suggesting that the observed ultrasensitivity did not arise from the zero-order effect rather than from the competition between Wee1 and alternative Cdk1 substrates.

To describe competition effects in phosphorylation/dephosphorylation cycles Ferrell and $\mathrm{Ha}$ proposed a model (Ferrell Jr. and Ha, 2014) where two substrates (X and $\mathrm{Y})$ are phosphorylated by a single kinase (K) according to a Michaelis-Menten scheme whereas dephosphorylation was assumed to occur by an auxiliary phosphatase in a first-order process (Fig. 10A). Interestingly, the analysis of this system yields a cubic steady state equation for the phosphorylation level of the low-affinity substrate that is structurally similar to Eq. (35) (Straube, 2015). Hence, one can expect the same type of input-output behaviors as in the case of receptor-ligand binding, i.e. hyperbolic, threshold-hyperbolic and ultrasensitive responses. To analyze a particular limiting regime one may use the approximations derived in Eqs. (37) and (38), and substitute the parameters in these equations by those corresponding to covalent modification cycles (cf. Table 1).

For example, in phosphorylation/dephosphorylation cycles the limit of strong substrate competition is defined by

$$
\varepsilon \cdot \beta \ll 1
$$

where $\varepsilon=K_{2} / K_{1}$ represents the ratio between the MichaelisMenten constants of the two substrates and

$$
\beta=\frac{\alpha_{2}}{1+\alpha_{2}} \frac{1+\alpha_{1}}{\alpha_{1}}, \quad \alpha_{i}=\frac{k_{-i}}{k_{i}}, \quad i=1,2
$$

represents a scale factor that involves ratios between the catalytic rates of phosphorylation and dephosphorylation of the two substrates (cf. Fig. 10A). Under the condition in Eq. (56) the concentration of the phosphorylation level of the lowaffinity substrate can be approximated by

$$
[X P] \approx\left\{\begin{array}{cc}
\varepsilon \beta \frac{X_{T}}{1+\alpha_{1}} \frac{K_{T}}{K_{T}^{*}-K_{T}}, & K_{T}<K_{T}^{*} \\
{[X P]_{-},} & K_{T}>K_{T}^{*}
\end{array}\right.
$$

where

$$
K_{T}^{*}=\frac{\alpha_{2}}{1+\alpha_{2}} Y_{T}
$$

defines a threshold concentration and $[X P]_{-}$is given by the negative root of the LR-type equation (cf. Eq. 38)

$$
x^{2}-\left(\frac{K_{T}-K_{T}^{*}}{\alpha_{1}}+\frac{X_{T}+K_{1}}{1+\alpha_{1}}\right) x+\frac{K_{T}-K_{T}^{*}}{\alpha_{1}} \frac{X_{T}}{1+\alpha_{1}}=0 .
$$

Using the parameter substitutions in Table 1 one can predict that the response curve increases hyperbolically beyond the threshold if $K_{1} \gg X_{T}$ whereas an ultrasensitive reponse requires $K_{2} \ll K_{1} \ll X_{T}$. Compared to the case of receptor-ligand binding both the threshold and the maximal phosphorylation level of the low-affinity substrate depend on the kinetic parameters of phosphorylation and dephosphorylation. Specifically, increasing $\alpha_{2}$ increases the threshold while increasing $\alpha_{1}$ lowers the maximal phosphorylation level (Fig. 10B).

\subsection{Bifunctional converter enzymes}

In some cases the activities of the two opposing enzymes in a covalent modification cycle are located on the same protein in which case modification and demodification are carried out by a 


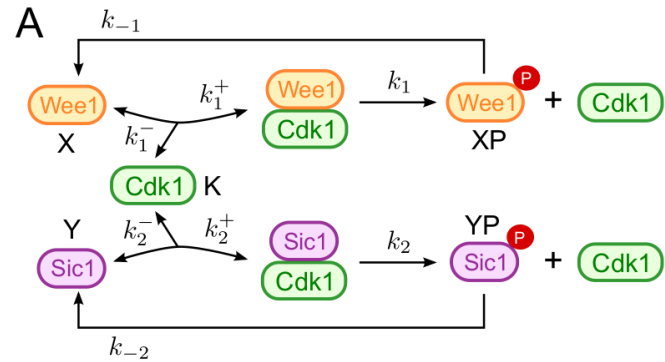

B

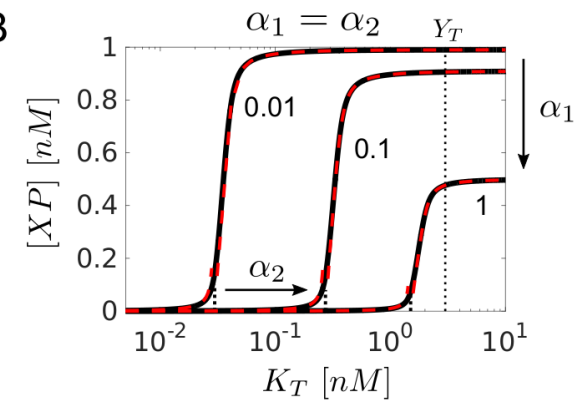

Figure 10: Substrate competition in phosphorylation/dephosphorylation cycles. (A) Two substrates ( $\mathrm{X}$ and $\mathrm{Y}$ ) compete for access to Cdk1 (K) which mediates phosphorylation of Wee1 and Sic1. Dephosphorylation is assumed to follow pseudo first-order kinetics. (B) Steady state concentration of the phosphorylated form of the low-affinity substrate as a function of the kinase concentration in the ultrasensitive regime. As $\alpha_{1}=k_{-1} / k_{1}$ and $\alpha_{2}=k_{-2} / k_{2}$ are simultaneously increased from 0.01 to 1 the threshold $K_{T}^{*}=\alpha_{2} Y_{T} /\left(1+\alpha_{2}\right)$ (short dotted lines) increases and the maximal amplitude $[X P]_{\max }=X_{T} /\left(1+\alpha_{1}\right)$ decreases while the steepness of the response curve remains the same. Red dashed lines were computed from Eq. (57). Parameters: $K_{1}=0.1 n M, K_{2}=10^{-3} n M$, $X_{T}=1 n M, Y_{T}=1 n M, k_{1}=k_{2}=1 / s$.

\begin{tabular}{lll|}
\hline $\begin{array}{l}\text { receptor-ligand } \\
\text { binding }\end{array}$ & $\begin{array}{l}\text { covalent } \\
\text { cycles }\end{array}$ & $\begin{array}{l}\text { modification } \\
\text { two-component } \\
\text { systems }\end{array}$ \\
\hline \hline$[L 1 . R]$ & {$[X P]$} & {$\left[R R 1^{*}\right]$} \\
\hline$R_{T}$ & $\frac{K_{T}}{\alpha_{1}}$ & $C_{p, 1}$ \\
\hline$L 2_{T}$ & $\frac{\alpha_{2}}{\alpha_{1}} \frac{Y_{T}}{1+\alpha_{2}}$ & $\frac{C_{p, 1}}{C_{p, 2}} R R_{2 T}$ \\
\hline$L 1_{T}$ & $\frac{X_{T}}{1+\alpha_{1}}$ & $R R_{1 T}$ \\
\hline$K_{d 1}$ & $\frac{K_{1}}{1+\alpha_{1}}$ & $C_{t, 1}$ \\
\hline$\frac{K_{d 2}}{K_{d 1}}$ & $\frac{K_{2}}{K_{1}} \frac{\alpha_{2}}{1+\alpha_{2}} \frac{1+\alpha_{1}}{\alpha_{1}}$ & $\frac{C_{p, 1}}{C_{p, 2}} / \frac{C_{t, 1}}{C_{t, 2}}$ \\
\hline
\end{tabular}

Table 1: Parameter substitutions for systems with substrate competition as described by Eq. (35). single bifunctional enzyme. Prominent examples are the isocitrate dehydrogenase kinase/phosphatase (IDHKP) involved in the regulation of the tricarboxylic acid cycle (LaPorte and Koshland Jr., 1982), the uridylyltransferase involved in nitrogen assimilation in E. coli (Garcia and Rhee, 1983; Ninfa et al., 2000) and the mammalian 6-phosphofructo-2-kinase/fructose2,6-bisphosphatase which integrates multiple signals to regulate the switch between glycolysis and gluconeogenesis in the liver (Kurland and Pilkis, 1995; Dasgupta et al., 2014).

Originally, it was believed that bifunctional enzymes mediate graded responses and robustness rather than ultrasensitivity (Ortega et al., 2002; Shinar et al., 2007, 2009; Hart et al., 2011a,b; Dexter and Gunawardena, 2013). The latter property can be readily understood from the observation that the enzyme concentration cancels out from the steady state equation (Eq. 44) because it appears in the forward $\left(K_{T}\right)$ as well as in the backward rate $\left(P_{T}\right)$. Hence, for bifunctional enzymes (with $\left.K_{T}=P_{T}\right)$ the parameter $\alpha=k_{1} K_{T} /\left(k_{2} P_{T}\right)=k_{1} / k_{2}$ becomes independent of the enzyme concentration so that changes in the latter would not affect the stimulus-response curves in Fig. 8. This form of concentration robustness has been related to structural properties of a network (Shinar and Feinberg, 2010) or to the existence of certain algebraic invariants (Dexter et al., 2015). More generally, robustness properties can be understood in terms of parameter non-identifiability (Sontag, 2017).

Concentration robustness may not only occur with respect to the enzyme concentration, but also to that of substrates and/or effectors. Interestingly, this can happen already at the level of the Goldbeter-Koshland model in the signal-transducing regime. To see this more explicitly, we rewrite the approximation for the stimulus-response curve (Fig. 8D) as a function of the substrate concentration, i.e.

$$
\left[S^{*}\right] \approx\left\{\begin{array}{cc}
S_{T}\left(1-\frac{K_{1}}{\alpha K_{2}-S_{T}}\right), & S_{T}<\alpha K_{2} \\
\alpha K_{2}, & S_{T}>\alpha K_{2}
\end{array} .\right.
$$

Hence, if $S_{T}>\alpha K_{2}$ the concentration of the phosphorylated substrate becomes independent of the substrate concentration $\left(\left[S^{*}\right] \approx \alpha K_{2}\right)$ so that $\left[S^{*}\right]$ exhibits concentration robustness with respect to $S_{T}$ in that regime.

Yet, there were known examples of demonstrated ultrasensitivity for bifunctional enzymes (Fig. 11A) raising the question how one can understand the emergence of ultrasensitivity in bifunctional enzyme systems (Straube, 2012). In fact, the first experimental evidence for enhanced sensitivity in covalent modification cycles came from studies with the bifunctional IDHKP (LaPorte and Koshland Jr., 1983) although the measured stimulus-response curve (Fig. 11B) more resembled that of a linear-saturation response as it occurs in the signaltransducing regime (Fig. 8D). Interestingly, both behaviors can be understood within a common mechanistic model which was originally proposed by Shinar et al. (Shinar et al., 2009) to describe IDHKP-mediated robustness in glyoxylate bypass regulation (LaPorte et al., 1985). Later, it was shown by means of a rapid equilibrium approximation that the Shinar model yields a GK-type equation (Straube, 2013) if one replaces in Eq. (44) Michaelis-Menten constants by dissociation constants. Hence, 
A

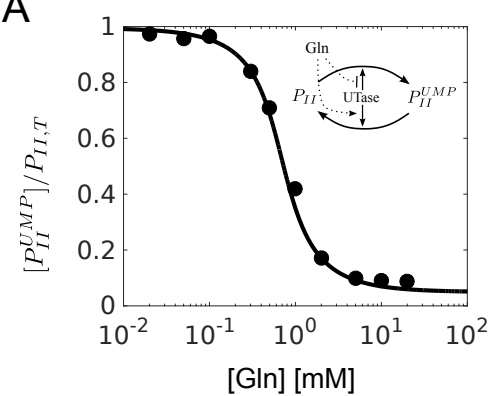

B

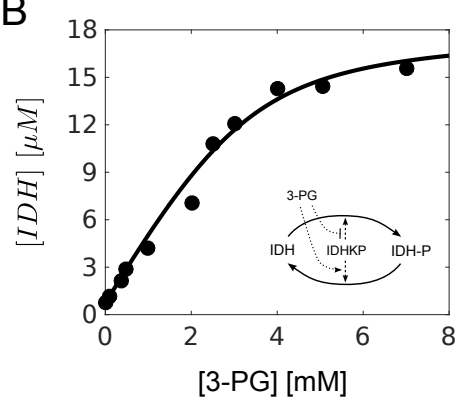

C

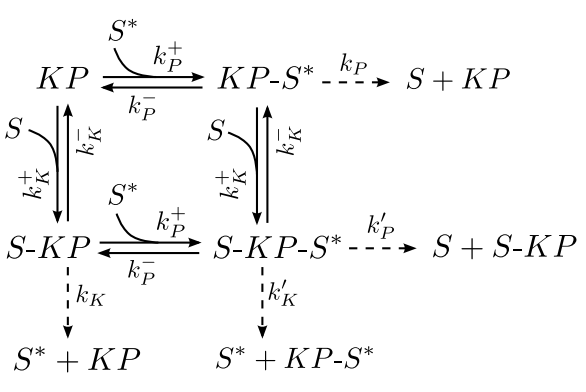

Figure 11: Ultrasensitivity in covalent modification cycles with a bifuctional converter enzyme. (A) Ultrasensitive response in the uridylylation of the PII protein (also known as GlnK) by the uridylyltransferase (UTase) which is reciprocally regulated by glutamine (Gln). (B) Isocitrate dehydrogenase (IDH) exhibits a linearsaturation response as a function of 3-PG which reciprocally regulates the isocitrate dehydrogenase kinase/phosphatase (IDHKP). Circles represent experimental results from Ref. (Ventura et al., 2010) (A) and (LaPorte and Koshland Jr., 1983) (B). Solid lines were computed based on the reaction network depicted in panel C using experimentally measured values (Straube, 2013). (C) Reaction mechanism for the phosphorylation of a substrate $\mathrm{S}$ by a bifunctional enzyme with two-catalytic sites according to Ref. (Shinar et al., 2009).

if a bifunctional enzyme exhibits two distinct catalytic sites for its opposing activities one may expect similar types of stimulusresponse curves as for covalent modification cycles with distinct converter enzymes (Fig. 8) including ultrasensitivity and concentration robustness where the latter occurs in the signaltransducing regime (Eq. 58). In support of this expectation evidence for concentration robustness has recently been observed in the regulation of cellular Pi homeostasis by PPIP5K which denotes a family of bifunctional enzymes with two domains for its kinase and phosphatase activities (Gu et al., 2017).

\subsubsection{Bifunctional enzymes with a single catalytic site}

Although many bifuctional enzymes seem to exhibit distinct catalytic sites for their opposing activities there are examples where modification and demodification are carried out by a single catalytic center. The most prominent members of this class are sensor histidine kinases which are part of two-component systems (cf. section 4). Based on the crystal structure it seems that also the IDHKP exhibits just a single catalytic site (Zheng and Jia, 2010) which would prevent formation of a ternary $S-K P-S^{*}$ complex as assumed in the Shinar model (Fig. 11C). This leads to the strange situation that, though the predictions based on this model quantitatively agree with the measured stimulus-response curve (Fig. 11B) it mechanistically does not apply to the case of the IDHKP. However, a revised model taking into account the structure of the active site as well as substrate dimerization confirms the existence of concentration robustness for the IDH cycle (Dexter and Gunawardena, 2013).

Apart from concentration robustness one might ask whether bifunctional enzymes with a single catalytic site may also yield ultrasensitivity? It turns out that this is, indeed, the case if one assumes that the opposing activities are reciprocally regulated by an allosteric effector (Fig. 12A). To this end, we assume that the enzyme may exist in a kinase state $\left(E_{K}\right)$ and in a phosphatase state $\left(E_{P}\right)$, and that binding of the effector to the kinase state switches it from kinase to phosphatase mode. Interestingly, the response behavior of such a system depends on the affinity of the effector (Straube, 2014). If the affinity is low $\left(K_{d} \gg E_{T}\right)$ the concentration of the phosphorylated form of the substrate exhibits a hyperbolic dependence on the effector concentration as described by

$$
\left[S^{*}\right] \approx \frac{S_{T}}{1+\frac{k_{2} / K_{2}}{k_{1} / K_{1}} \frac{L_{T}}{K_{d}}} .
$$

Here, $K_{1}$ and $K_{2}$ denote respectively the Michaelis-Menten constants associated with the kinase and the phosphatase activity, and $K_{d}=k_{d}^{-} / k_{d}^{+}$denotes the dissociation constant of the enzyme-effector complex. In contrast, if the enzyme exhibits a high affinity for the effector $\left(K_{d} \ll E_{T}\right)$ the steady state is determined a GK-type equation of the form

$$
k_{1}\left(E_{T}-L_{T}\right) \frac{S_{T}-\left[S^{*}\right]}{K_{1}+S_{T}-\left[S^{*}\right]} \approx k_{2} L_{T} \frac{\left[S^{*}\right]}{K_{2}+\left[S^{*}\right]}
$$

where $E_{T}-L_{T}$ and $L_{T}$ play the role of an effective kinase and phosphatase concentration, respectively. In this regime bifunctional enzymes with a single catalytic site may generate similar response behaviors as covalent modification cycles with distinct converter enzymes. However, this regime is restricted to low effector concentration $\left(L_{T}<E_{T}\right)$ where the enzyme is not saturated by the effector.

\section{Two-Component Signal Transduction}

Two-component systems (TCSs) are modular signal transduction systems which are utilized by bacteria and other microbes to sense and respond to diverse stimuli (Stock et al., 2000) such as osmolarity (EnvZ/OmpR), magnesium limitation (PhoQ/PhoP) or nitrogen assimilation (NtrB/NtrC). While $E$. coli comprises about 30 of these systems, other bacteria express more than 100 TCSs (Alm et al., 2006). Canonical TCSs consist of a sensor histidine kinase (HK) and a cognate response regulator (RR) which often acts as a transcription factor to activate or repress a particular set of response genes. The HK exhibits up to three distinct activities to modulate the RR phosphorylation level: Upon stimulation, the HK autophosphorylates at a conserved histidine residue and transfers the phosphoryl group to an aspartate residue in the RR receiver domain. The 
A
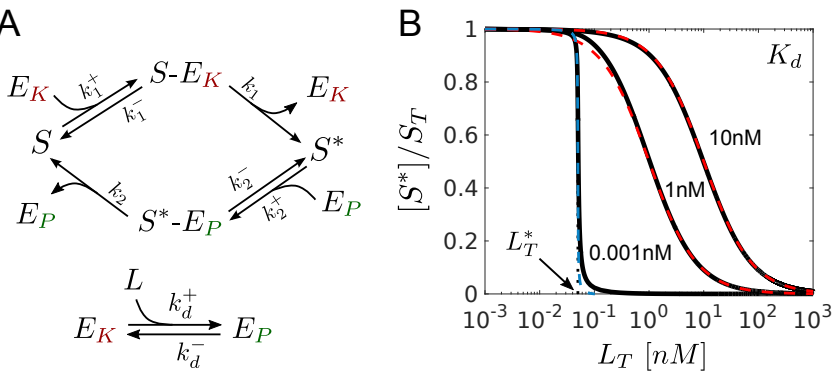

Figure 12: Reciprocal regulation in covalent modification cycles with a bifunctional converter enzyme. (A) Reaction mechanism for an enzyme with a single catalytic site. $E_{K}$ and $E_{P}$ denote enzyme states in kinase and phosphatase mode, respectively. Binding of a ligand $L$ to $E_{K}$ is assumed to switch the enzyme into phosphatase mode. (B) Stimulus-response curves for increasing binding affinity. At low affinity $K_{d} \gg E_{T}\left(K_{d}=k_{d}^{-} / k_{d}^{+}\right)$the response is graded, at high affinity $\left(K_{d} \ll E_{T}\right)$ the response becomes ultrasensitive, but is restricted to $L_{T}<E_{T}$. Dashed curves were computed from Eqs. (59) (red) and (60) (blue). Parameters: $E_{T}=0.1 n M, S_{T}=10 n M, K_{1}=K_{2}=0.1 n M, k_{1}=k_{2}=1 / s$.

unphosphorylated form of the HK often exhibits a distinct phosphatase activity towards the phosphorylated form of RR endowing many HKs with a bifunctional design (Goulian, 2010).

\subsection{The Batchelor-Goulian Model}

More than 20 years ago Russo and Silhavy argued that if HK autophosphorylation represents the rate-limiting step in RR phosphorylation TCSs with a bifunctional design should exhibit special robustness properties. Specifically, the RR phosphorylation level should be approximately independent with respect to variations in both the concentration of the $\mathrm{HK}$ and that of the RR (Russo and Silhavy, 1993). Ten years later, by measuring the transcriptional activity of OmpR-regulated genes, Batchelor and Goulian provided the first experimental evidence for concentration robustness in TCSs (Batchelor and Goulian, 2003). They also proposed a simple model that could qualitatively account for the observed robustness under variations of $\mathrm{RR}$ and HK abundances (Fig. 13A). Under conditions when the HK is much less abundant than the RR (which is the typical situation in TCSs) the steady states within the Batchelor-Goulian model are determined by the LR-type equation (cf. Eq. 8)

$$
\left[R R^{*}\right]^{2}-\left(C_{p}+R R_{T}+C_{t}\right)\left[R R^{*}\right]+C_{p} R R_{T} \approx 0
$$

where $R R^{*}$ stands for the phosphorylated form of the RR,

$$
C_{p}=\frac{k_{K}^{+}}{k_{p}} K_{p} \quad \text { and } \quad C_{t}=\frac{k_{K}^{-}}{k_{t}} K_{t}
$$

denote rescaled Michaelis-Menten constants associated with the phosphatase and the kinase activities of the $\mathrm{HK}$, and $R R_{T}$ denotes the total RR concentration. In the 'high-affinity' limit, defined by $C_{t} \ll C_{p}$, the solution of Eq. (61) can be approximated by

$$
\left[R R^{*}\right] \approx\left\{\begin{array}{cc}
R R_{T}, & R R_{T}<C_{p} \\
C_{p}, & R R_{T}>C_{p}
\end{array},\right.
$$

i.e. if the concentration of the RR exceeds the threshold $C_{p}$ its level remains approximately constant. Note that the value
A
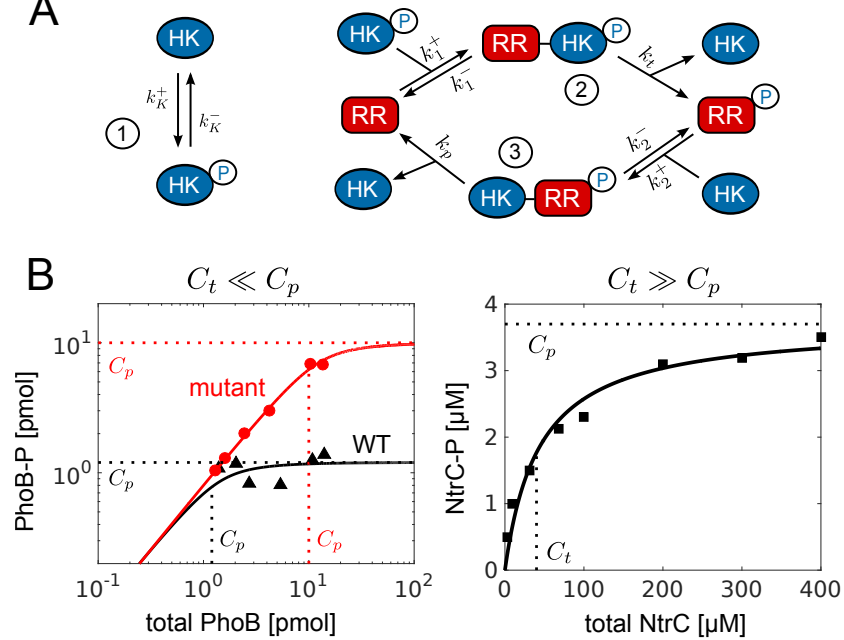

Figure 13: Concentration robustness in the Batchelor-Goulian model. (A) Reaction scheme includes HK autophosphorylation (1), phosphotransfer (2) and HK phosphatase activity (3). (B) Comparison of the Batchelor-Goulian model with measurements of the RR phosphorylation level as a function of total RR abundance. Left panel: PhoR/PhoB system (Gao and Stock, 2013). The mutant PhoB exhibits reduced affinity for PhoR which increases the value of $C_{p}$. The stimulus-response curves are of linear-saturation type as predicted by Eq. (63). Right panel: NtrB/NtrC system (Jiang et al., 2012). The stimulus-response curve exhibits a Michaelian shape as predicted by Eq. (64).

of this constant $\left(C_{p}\right)$ only depends on kinetic parameters, but not on protein concentrations (Eq. 62). In the 'low-affinity' regime, defined by $C_{t} \gg C_{p}$, the solution of Eq. (61) can be approximated by

$$
\left[R R^{*}\right] \approx \frac{C_{p} R R_{T}}{C_{p}+R R_{T}+C_{t}} .
$$

Interestingly, both types of behaviors were observed experimentally (Fig. 13B). The Batchelor-Goulian model has also been used to model other regulatory aspects of TCSs such as the impact of autoregulation (Miyashiro and Goulian, 2008) and the emergence of cross-talk (Siryaporn et al., 2010). However, there also exist alternative TCS models which describe, for example, phosphotransfer as a reversible reaction (Kremling et al., 2004; Tindall et al., 2013) or include the formation of dead-end complexes (Igoshin et al., 2008).

\subsection{Reciprocal regulation of $H K$ activities}

A quantitative analysis of the input-output behavior of TCSs is often limited by the fact that the physiological signal sensed by the HK is not well known (Krell et al., 2010). In cases where the signal has been identified it became evident that the HK's autokinase and phosphatase activities are the primary targets of regulation (Stewart, 2010; Heermann and Jung, 2010) and that regulation of these activities often occurs in a reciprocal manner (Jiang and Ninfa, 1999; Chamnongpol et al., 2003; Dubey et al., 2016). Figure 14A shows an example where binding of an allosteric ligand switches the HK from kinase to phosphatase mode, i.e. in the presence of the signal the system is shut-off and only when the signal becomes limiting the system is switched on. Similar regulatory structures have been 
observed in the PhoQ/PhoP and NtrB/NtrC systems (Jiang and Ninfa, 1999; Chamnongpol et al., 2003).

Similar as in the case of covalent modification cycles with a bifunctional converter enzyme (cf. Fig. 12) the steady state behavior of a TCS with reciprocal regulation depends on the affinity of the ligand. If the affinity is low $\left(K_{d} \gg H K_{T}\right)$ the steady state equation becomes identical with the LR-type equation in Eq. (61) if $C_{p}$ is replaced by $C_{p}^{*}=C_{p} K_{d} / L_{T}$ where $L_{T}$ denotes the total ligand concentration and $K_{d}=k_{d}^{-} / k_{d}^{+}$denotes the dissociation constant for ligand binding. Hence, in this regime the threshold for reaching a constant phosporylation level as well as the value of this constant depend on the ligand concentration (Fig. 14B, left panel), i.e. the system exhibits stimulus-dependent concentration robustness (Straube, 2014). In contrast, if the ligand exhibits a high affinity for the HK the steady state is determined by a GK-type equation

$$
k_{t}^{a p p}\left(H K_{T}-L_{T}\right) \frac{R R_{T}-\left[R R^{*}\right]}{K_{t}^{a p p}+R R_{T}-\left[R R^{*}\right]} \approx k_{p} L_{T} \frac{\left[R R^{*}\right]}{K_{p}+\left[R R^{*}\right]}
$$

where $K_{P}$ denotes the Michaelis-Menten constant associated with the phosphatase activity of the HK and

$$
k_{t}^{a p p}=\frac{k_{t}}{1+k_{t} / k_{K}^{+}} \quad \text { and } \quad K_{t}^{a p p}=K_{t} \frac{1+k_{K}^{-} / k_{K}^{+}}{1+k_{t} / k_{K}^{+}}
$$

denote apparent constants that can be expressed in terms of rate constants and the Michaelis-Menten constant associated with the phosphotransferase activity $\left(K_{t}\right)$. Hence, when triggered by a high-affinity ligand TCSs may behave similarly to covalent modification cycles and exhibit ultrasensitivity to input signals (Fig. 14B, right panel). In this regime TCSs may even exhibit bistability if ultrasensitivity is combined with a positive feedback loop, e.g. due to autoregulation of the HK and RR genes (Wei et al., 2014). However, as can be seen from Eq. (65) the occurrence of ultrasensitivity is restricted to ligand concentrations that are lower than that of the sensor kinase (such that $H K_{T}-L_{T}>0$ ) making this operating regime particularly useful to detect and amplify low-abundant input signals.

\subsection{Cross-talk}

New input-output functionality of TCSs is often acquired through gene duplication and subsequent divergence of paralogous genes (Alm et al., 2006; Capra et al., 2012). As a result, many sensor kinases do not only phosphotransfer to their cognate response regulator, but (on longer time scales) also to those of other TCSs (Fisher et al., 1996; Skerker et al., 2005). However, in vivo cross-talk from a HK to a non-cognate RR seems to be extremely rare being only observable after the cognate RR and the non-cognate HK have been genetically eliminated (Fig. 15A) (Siryaporn and Goulian, 2008; Groban et al., 2009). To ensure this high degree of specificity three main mechanisms have been identified: molecular recognition, phosphatase activity of the HK and substrate competition (Podgornaia and Laub, 2013). Among these mechanisms molecular recognition appears to be the most dominant one. Experiments have shown that the specificity constant of a $\mathrm{HK}$ as measured by the $k_{c a t} / K_{M}$
A
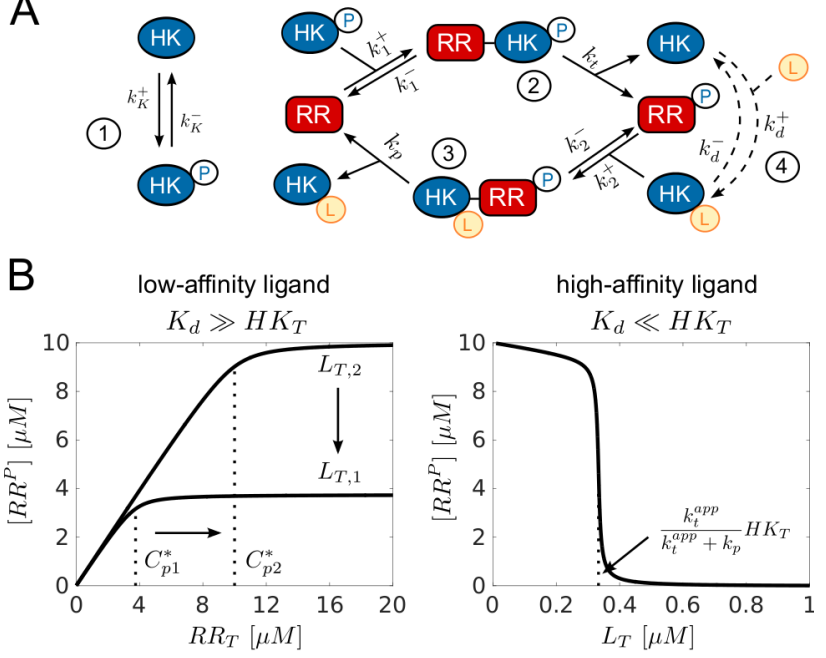

Figure 14: Reciprocal regulation in two-component systems. (A) Binding of a ligand (4) inhibits autophosphorylation (1) and phosphotransfer (2), but activates the HK's phosphatase activity (3). (B) Depending on the binding affinity of the ligand $\left(K_{d}=k_{d}^{-} / k_{d}^{+}\right)$the system may either exhibit concentration robustness (left panel) or ultrasensitivity (right panel).

ratio is $10^{4}-10^{5}$ times higher for the cognate RR (Fisher et al., 1996; Grimshaw et al., 1998; Skerker et al., 2005) implying a huge kinetic preference of a HK for its cognate RR. In fact, mathematical modeling has suggested that there is a strong selective pressure to eliminate cross-talk between TCSs after gene duplication before new input-output functionality can be acquired (Rowland and Deeds, 2014).

To understand how a strong kinetic preference may generate pathway insulation within the Batchelor-Goulian model we consider the case where one HK phosphorylates its cognate partner (RR2) and a non-cognate regulator denoted by RR1 (Fig. 15B and C). The steady state equation for the phosphorylated form of RR1 is a cubic polynomial that is structurally similar to Eq. (35) (cf. Supporting Information and Table 1). Its steady state behavior depends on whether the HK exhibits a kinetic preference (for RR2) with respect to its phosphotransferase activity $\left(\varepsilon_{t} \gg 1\right)$ or with respect to its phosphatase activity $\left(\varepsilon_{p} \gg 1\right)$ where $\varepsilon_{t}$ and $\varepsilon_{p}$ are defined by (cf. Fig. 15C)

$$
\varepsilon_{t}=\frac{k_{t, 2} / K_{t, 2}}{k_{t, 1} / K_{t, 1}} \quad \text { and } \quad \varepsilon_{p}=\frac{k_{p, 2} / K_{p, 2}}{k_{p, 1} / K_{p, 1}} .
$$

In the first case $\left(\varepsilon_{t} \gg 1\right)$ the steady states for $\left[R R_{2}^{*}\right]$ and $\left[R R_{1}^{*}\right]$ are determined (to leading order) by the LR-type equations

$$
\left[R R_{2}^{*}\right]^{2}-\left[R R_{2}^{*}\right]\left(R R_{2 T}+C_{p, 2}+C_{t, 2}\right)+C_{p, 2} R R_{2 T} \approx 0
$$

and

$$
\left[R R_{1}^{*}\right]^{2}-\left(R R_{1 T}+C_{p, 1}^{\mathrm{eff}}+C_{t, 1}\right)\left[R R_{1}^{*}\right]+C_{p, 1}^{\mathrm{eff}} R R_{1 T} \approx 0
$$

where $C_{p, 1}^{\text {eff }}=C_{p, 1}-\varepsilon_{p} R R_{2 T}$. Hence, the cognate RR (RR2) behaves as if the non-cognate partner was absent while the latter may exhibit threshold behavior if $C_{t, 1} \ll R R_{1 T}$. For autophosphorylation rates below a threshold the phosphorylation level 
of $R R_{1}^{*}$ remains low, too, i.e.

$$
\left[R R_{1}^{*}\right] \approx \frac{1}{\varepsilon_{t}} \frac{R R_{1 T} C_{p, 1}}{R R_{2 T}-C_{p, 2}}, \quad k_{K}^{+}<\frac{k_{p, 2}}{K_{p, 2}} R R_{2 T},
$$

beyond the treshold it increases either hyperbolically $\left(C_{t 1} \gg\right.$ $\left.R R_{1 T}\right)$ or in an ultrasensitive manner $\left(C_{t 1} \ll R R_{1 T}\right)$ (Fig. 15D).

In contrast, if the HK develops a kinetic preference with respect to its phosphatase activity $\left(\varepsilon_{p} \gg 1\right)$ the steady state for $\left[R R_{2}^{*}\right]$ is still determined by Eq. (66) while that for $\left[R R_{1}^{*}\right]$ is determined by a different LR-type equation

$$
\left[R R_{1}^{*}\right]^{2}-\left(R R_{1 T}+C_{p, 1}+C_{t, 1}^{\mathrm{eff}}\right)\left[R R_{1}^{*}\right]+C_{p, 1} R R_{1 T} \approx 0
$$

where $C_{t, 1}^{\mathrm{eff}}=C_{t, 1}+\varepsilon_{t} R R_{2 T}$. In that case no threshold exists and the order in which the RRs are phosphorylated is reversed. As a result the non-cognate RR becomes fully phosphorylated already at low autophosphorylation rates (Fig. 15E). Together this suggests that to insulate newly emerging TCSs developing a kinetic preference with respect to the phosphotransferase rather than the phosphatase activitity should be the better strategy which is supported by the fact that the latter has not been observed experimentally yet (Siryaporn and Goulian, 2008).

In some cases cross-talk where two RRs are phosphorylated by a single HK may be a desirable feature of a regulatory network in which case it has been referred to as cross-regulation (Laub and Goulian, 2007). For example, in the chemotaxis network of E. coli the histidine kinase CheA can phosphorylate either of the two regulator proteins $\mathrm{CheY}$ and $\mathrm{CheB}$ : while CheY$\mathrm{P}$ controls the sense of rotation of the flagellar motor CheB$\mathrm{P}$ mediates feedback regulation at the receptor level (Sourjik, 2004). Also, since the threshold for the non-cognate partner depends on the concentration of the cognate RR (Eq. 68) the 1HK/2RR motif (Fig. 15B) has been suggested as a basis for constructing a tunable threshold device in the context of synthetic biology applications (Amin et al., 2014).

\section{Conclusions}

In physics, the superior importance of the harmonic oscillator derives from its repeated occurrence in different branches of physics which allows describing diverse phenomena such as vibrational modes in solid states or oscillations in electrical circuits using similar equations. In this review we advocated the opinion that, as far as the steady state behavior is concerned, elementary network motifs might play a similar role for understanding the behavior of larger biological networks. We have illustrated this approach starting from a simple bimolecular reaction and adding successively more complexity such as substrate competition, cooperativity or covalent modifications. If analyzed in the limit of strong or weak coupling (e.g. high or low affinity of a ligand) or under conditions of substrate excess the corresponding steady state equations became structurally similar to those of a bimolecular reaction (L-type and LR-type equations) or to that of the Goldbeter-Koshland model describing covalent modifications (GK-type equation).
A

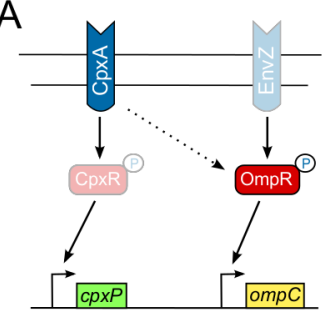

B
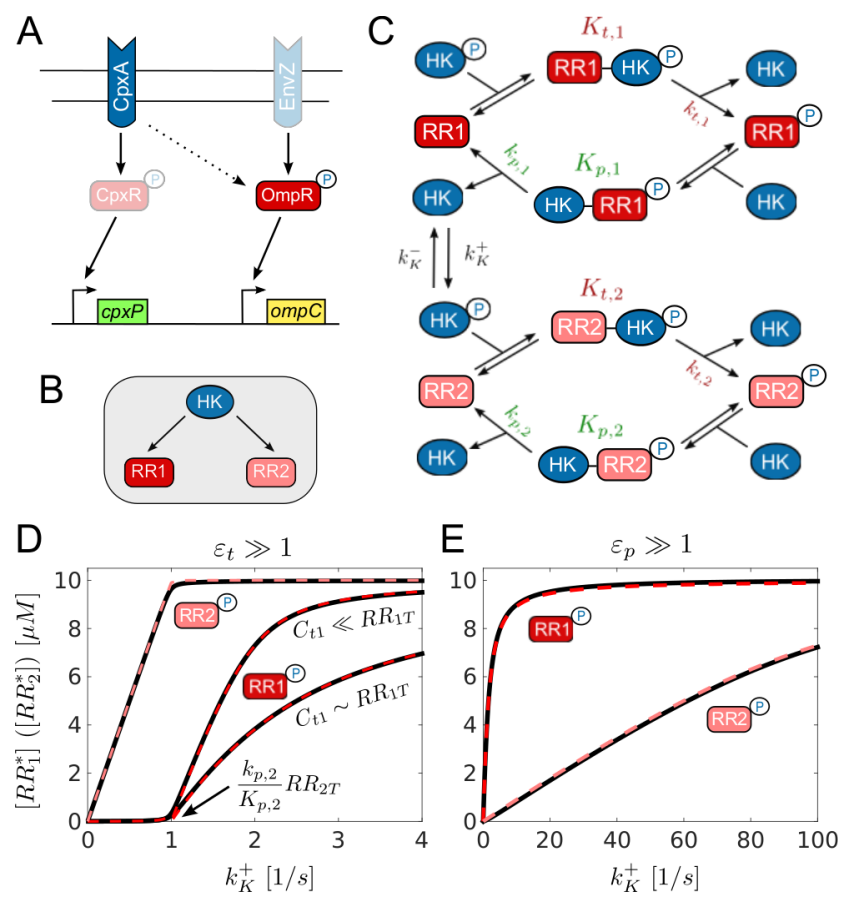

Figure 15: Cross-talk and substrate competition in two-component systems. (A) In vivo, cross-talk from CpxA to OmpR is only observable in the absence of CpxR and EnvZ (Siryaporn and Goulian, 2008). (B) Cross-talk motif: 1HK phosphorylates to RRs. (C) Detailed model for the motif in B. (D) If the HK exhibits a kinetic preference for RR2 with respect to its phosphotransferase activity $\left(\varepsilon_{t} \gg 1\right) \mathrm{RR} 2$ becomes insulated and $\left[R R_{1}^{*}\right]$ exhibits a threshold in the response curve. Parameters: $K_{p, 1}=K_{p, 2}=1 \mu M, k_{p, 1}=k_{p, 2}=k_{t, 1}=0.1 / \mathrm{s}$, $k_{t, 2}=1 / s$. Lower curve: $K_{t, 1}=10 \mu M, K_{t, 2}=0.1 \mu M$. Upper curves: $K_{t, 1}=$ $1 \mu M, K_{t, 2}=0.01 \mu M$. Dashed curves were computed from Eqs. (66), (67) and (68). (E) If the HK exhibits a kinetic preference for RR2 with respect to its phosphatase activity $\left(\varepsilon_{p} \gg 1\right)$ RR2 becomes insulated, but the non-cognate RR (RR1) becomes phosphorylated first. Parameters: $K_{t, 1}=K_{t, 2}=1 \mu M$, $k_{t, 1}=k_{t, 2}=k_{p, 1}=0.1 / s, k_{p, 2}=1 / s, K_{p, 1}=1 \mu M, K_{p, 2}=0.1 \mu M$. Dashed curves were computed from Eqs. (69) and (66). Other parameters: $R R_{1 T}=$ $R R_{2 T}=10 \mu M, k_{K}^{-}=0.1 / s$ 
Different limits may lead to different equations as in the case of two-component systems where the sensor kinase is reciprocally regulated by an allosteric effector (Fig. 14): If the affinity of the effector is high the system is described by an equation of the Goldbeter-Koshland type (Eq. 65); in the low-affinity limit it becomes similar to that of a bimolecular reaction (Eq. 61). In these equations the parameters of the elementary motifs are typically replaced by parameters with a different meaning or by parameter combinations. In the latter case information from different parts of the network is condensed into few effective parameters (cf. Table 1) which allows for a quantitative understanding of signal integration.

Despite the fact that different systems can be described by similar equations the interpretation of the resulting input-output behavior might be different. For example, under conditions of substrate excess two-component systems can be described by a similar equation as receptor-ligand binding. However, for twocomponent systems the receptor concentration is replaced by a rescaled Michaelis-Menten constant which only depends on kinetic parameters of the system. As a consequence the stimulusresponse curve for receptor-ligand binding describes saturation of a receptor by a ligand while that for a two-component system describes concentration robustness (Fig. 13).

\subsection{Challenges and future directions}

In this review we focussed on the simplest network motifs within each class, e.g. two ligands competing for a binding site on a receptor, for which analytical results are known. However, in vivo the number of competitors could be substantially higher which may lead to multiple thresholds and hierarchical regulation (Levine et al., 2007; Mitarai et al., 2007). As the number of competitors increases the polynomial order of the corresponding steady state equations increases, too. To systematically derive and analyze such equations algebraic approaches have proven to be useful (Thomson and Gunawardena, 2009; Feliu et al., 2012; Estrada et al., 2016). When going from 2 to $\mathrm{n}$ competitors or from one covalent modification cycle to $\mathrm{n}$ such cycles, as in a MAP kinase cascade, it will be interesting to see if the resulting steady state equations can still be reduced to a $\mathbf{L}$-type, LR-type or GK-type equation. If so, this may provide a classification of network architectures based on their decomposability into elementary network motifs. One could also envision a classification where larger networks, that can be described by the same equation as an elementary network motif, may be replaced by an 'equivalent circuit' of the motif with renormalized input-output parameters similar as for electrical circuits. Such a classification would be particularly useful to aid the construction and integration of novel regulatory circuits in the context of synthetic biology.

While this review almost exclusively focussed on steady state aspects of regulatory networks it remains a major challenge to decode the information processed by cells that is encoded in the temporal response of a network (Sasagawa et al., 2005; Thurley et al., 2012; Levine et al., 2013). For example, the physiological response may depend on whether a steady state is approached monotonically or through relaxation after an overshoot (Shin et al., 2006). In ODE systems with two or more state variables a steady state may become unstable in a Hopf bifurcation giving rise to sustained oscillations which encode information in their shape and their frequency. Similar as for steady states an approach based on network motifs was used to classify biological oscillators (Novak and Tyson, 2008). At least close to bifurcation points the transient dynamics of such systems can be described by 'normal form' equations which contain only a few effective parameters (Kuznetsov, 1998). Away from bifurcation points systems typically have to be analyzed on a case by case basis, and it remains an open question whether the transient dynamics of larger networks can be understood in terms of the known behavior of some elementary motifs. However, model reduction techniques such as the total QSSA may provide a suitable starting point for a systematic analysis.

\section{Appendix}

\subsection{Approximations for $\tau_{s}$}

If $K_{d} \ll\left|L_{T}-R_{T}\right|$ and $L_{T} \neq R_{T}$ we can approximate $\tau_{s}$, defined in Eq. (9), by

$$
\begin{aligned}
\tau_{s} & =\frac{K_{d}}{k_{d}^{-} \sqrt{\left(L_{T}-R_{T}\right)^{2}+2 K_{d}\left(L_{T}+R_{T}\right)+K_{d}^{2}}} \\
& \approx \frac{K_{d}}{k_{d}^{-}\left|L_{T}-R_{T}\right| \sqrt{1+2 \frac{K_{d}\left(L_{T}+R_{T}\right)}{\left(L_{T}-R_{T}\right)^{2}}}} \\
& \approx \frac{1}{k_{d}^{+}\left|L_{T}-R_{T}\right|}\left(1-\frac{K_{d}\left(L_{T}+R_{T}\right)}{\left(L_{T}-R_{T}\right)^{2}}\right)
\end{aligned}
$$

where we have used that $\sqrt{1+x} \approx 1+x / 2$ and $1 /(1+x) \approx 1-x$ when $x \ll 1$. For $L_{T}=R_{T}$ the leading order diverges and we obtain from Eq. (70)

$$
\begin{aligned}
\tau_{s} & =\frac{K_{d}}{k_{d}^{-} \sqrt{4 K_{d} R_{T}+K_{d}^{2}}}=\frac{1}{k_{d}^{-}} \frac{\sqrt{\frac{K_{d}}{4 R_{T}}}}{\sqrt{1+\frac{K_{d}}{4 R_{T}}}} \\
& \approx \frac{1}{k_{d}^{-}} \sqrt{\frac{K_{d}}{4 R_{T}}}\left(1-\frac{K_{d}}{8 R_{T}}\right) .
\end{aligned}
$$

In the limit $K_{d} \gg \max \left(L_{T}, R_{T}\right)$ we approximate $\tau_{s}$ by

$$
\begin{aligned}
\tau_{s} & =\frac{1}{k_{d}^{-} \sqrt{1+2 \frac{L_{T}+R_{T}}{K_{d}}+\left(\frac{L_{T}-R_{T}}{K_{d}}\right)^{2}}} \\
& \approx \frac{1}{k_{d}^{-}\left(1+\frac{L_{T}+R_{T}}{K_{d}}\right)}
\end{aligned}
$$

which agrees with the expression in Eq. (13).

\subsection{Approximations for the cooperative binding motif}

\subsubsection{Positive cooperativity}

In the limit of strong positive cooperativity $(c \gg b)$ we rewrite Eq. (27) in the form

$$
x^{2}-\left(K_{1 T}+K_{2 T}+\varepsilon S_{T}\right) x+(1+\varepsilon) K_{1 T} K_{2 T}=0
$$


where

$$
\varepsilon=\frac{b}{c-b} \ll 1
$$

For fixed $S_{T} \sim O\left(K_{1 T}, K_{2 T}\right)$ substituting the Ansatz

$$
x=x_{0}+\varepsilon x_{1}+O\left(\varepsilon^{2}\right)
$$

into Eq. (71) yields

$$
x_{0}^{2}-\left(K_{1 T}+K_{2 T}\right) x_{0}+K_{1 T} K_{2 T}=0
$$

and

$$
2 x_{0} x_{1}-\left(K_{1 T}+K_{2 T}\right) x_{1}-S_{T} x_{0}+K_{1 T} K_{2 T}=0 .
$$

Since $x \leq \min \left(K_{1 T}, K_{2 T}\right)$ the solution of Eq. (72) is given by

$$
\begin{aligned}
x_{0} & =\frac{K_{1 T}+K_{2 T}}{2}-\frac{\left|K_{1 T}-K_{2 T}\right|}{2} \\
& =\left\{\begin{array}{lc}
K_{1 T} & K_{1 T}<K_{2 T} \\
K_{2 T}, & K_{1 T}>K_{2 T}
\end{array} .\right.
\end{aligned}
$$

Substituting this solution into Eq. (73) and solving for $x_{1}$ gives

$$
x_{1}=\left\{\begin{array}{ll}
-K_{1 T} \frac{S_{T}-K_{2 T}}{K_{2 T}-K_{1 T}}, & K_{1 T}<K_{2 T}<S_{T} \\
-K_{2 T} \frac{S_{T}-K_{1 T}}{K_{1 T}-K_{2 T}}, & K_{2 T}<K_{1 T}<S_{T}
\end{array} .\right.
$$

Together, this yields the approximation

$$
x \approx \begin{cases}K_{1 T}\left(1-\varepsilon \frac{S_{T}-K_{2 T}}{K_{2 T}-K_{1 T}}\right), & K_{1 T}<K_{2 T}<S_{T} \\ K_{2 T}\left(1-\varepsilon \frac{S_{T}-K_{1 T}}{K_{1 T}-K_{2 T}}\right), & K_{2 T}<K_{1 T}<S_{T}\end{cases}
$$

which has been used to construct the solution in Eq. (31).

For large $S_{T}$ the term $\varepsilon S_{T}$ must not be neglected against $K_{1 T}$ and $K_{2 T}$ in the linear term of Eq. (71). Specifically, if $\varepsilon S_{T} \gg$ $K_{1 T}+K_{2 T}$ the linear term becomes dominant so that balancing it with the $O$ (1) part of the constant term yields the asymptotic form of the solution (cf. Eq. 29)

$$
x \sim \frac{K_{1 T} K_{2 T}}{K_{1 T}+K_{2 T}+\varepsilon S_{T}}, \quad S_{T} \gg \frac{K_{1 T}+K_{2 T}}{\varepsilon} .
$$

\subsubsection{Negative cooperativity}

In the case of strong negative cooperativity $(c \ll b)$ we rewrite Eq. (27) in the form

$$
x^{2}-\left(K_{1 T}+K_{2 T}-(1+\eta) S_{T}\right) x-\eta K_{1 T} K_{2 T}=0
$$

where

$$
\eta=\frac{c}{b-c} \ll 1 .
$$

Substituting the Ansatz

$$
x=x_{0}+\eta x_{1}+O\left(\eta^{2}\right)
$$

into Eq. (75) yields

$$
\left(x_{0}-K_{1 T}+K_{2 T}-S_{T}\right) x_{0}=0
$$

and

$$
2 x_{0} x_{1}-\left(K_{1 T}+K_{2 T}-S_{T}\right) x_{1}+S_{T} x_{0}-K_{1 T} K_{2 T}=0 .
$$

The non-negative solutions of Eq. (76) are given by

$$
\begin{aligned}
& x_{0}^{(1)}=0 \\
& x_{0}^{(2)}=K_{1 T}+K_{2 T}-S_{T}, \quad S_{T}<K_{1 T}+K_{2 T} .
\end{aligned}
$$

Substituting $x_{0}^{(i)}$ into Eq. (77) and solving for $x_{1}$ gives

$$
\begin{aligned}
& x_{1}^{(1)}=\frac{K_{1 T} K_{2 T}}{S_{T}-\left(K_{1 T}+K_{2 T}\right)}, \quad S_{T}>K_{1 T}+K_{2 T} \\
& x_{1}^{(2)}=-\left(S_{T}-\frac{K_{1 T} K_{2 T}}{K_{1 T}+K_{2 T}-S_{T}}\right), \quad S_{T}<K_{1 T}+K_{2 T} .
\end{aligned}
$$

Together, this yields the approximation (cf. Eq. 30)

$$
x \approx\left\{\begin{array}{cl}
\eta_{S_{T}-\left(K_{1 T}+K_{2 T}\right)}, & K_{1 T}<S_{T}-K_{2 T} \\
K_{1 T}+K_{2 T}-S_{T}, & K_{1 T}>S_{T}-K_{2 T}
\end{array}\right.
$$

which has been used to construct the solution in Eq. (32).

\subsection{Stimulus response curve for substrate competition at large substrate concentration}

The steady state for $[L 1 . R]$ is determined by the cubic equation in Eq. (35). To derive an approximate expression for the stimulus response curve in the limit $L 1_{T} \gg\left(R_{T}-L 2_{T}\right) / \varepsilon$ with $\varepsilon=K_{d 2} / K_{d 1} \ll 1$ we note that $[L 1 . R] \leq R_{T}$ remains bounded as $L 1_{T}$ becomes large so that the cubic term can be neglected in that limit. Then, keeping only the dominant terms from the coefficients of the linear and the quadratic terms, the stimulus response curve is determined to leading order by

$$
L_{1 T}[L 1 . R]^{2}-L 1_{T}\left(R_{T}-L 2_{T}-\varepsilon L 1_{T}\right)[L 1 . R]+\varepsilon R_{T} L 1_{T}^{2} \approx 0
$$

which agrees with Eq. (40).

Adler, M., Mayo, A., Alon, U., 2014. Logarithmic and power law input-output relations in sensory systems with fold-change detection. PLoS Comput. Biol. 10, e1003781.

Alm, E., Huang, K., Arkin, A., 2006. The evolution of two-component systems in bacteria reveals different strategies for niche adaptation. PLoS Comput. Biol. 2, e143.

Amin, M., Kothamachu, V. B., Feliu, E., Scharf, B. E., Porter, S. L., Soyer, O. S., 2014. Phosphate sink containing two-component signaling systems as tunable threshold devices. PLoS Comput Biol 10, e1003890.

Babel, H., Bischofs, I. B., 2016. Molecular and cellular factors control signal transduction via switchable allosteric modulator proteins (SAMPs). BMC Syst. Biol. 10, 35.

Batchelor, E., Goulian, M., 2003. Robustness and the cycle of phosphorylation and dephosphorylation in a two-component regulatory system. Proc. Natl. Acad. Sci. USA 100, 691-696.

Blüthgen, N., Bruggeman, F. J., Legewie, S., Herzel, H., Westerhoff, H. V., Kholodenko, B. N., 2006. Effects of sequestration on signal transduction cascades. FEBS J. 273, 895-906.

Borghans, J. A. M., de Boer, R. J., Segel, L. A., 1996. Extending the quasisteady state approximation by changing variables. Bull. Math. Biol. 58, 4363.

Borisov, N. M., Markevich, N. I., Hoek, J. B., Kholodenko, B. N., 2005. Signaling through receptors and scaffolds: independent interactions reduce combinatorial complexity. Biophys J 89, $951-966$.

Buchler, N. E., Louis, M., 2008. Molecular titration and ultrasensitivity in regulatory networks. J. Mol. Biol. 384, 1106-1119.

Capra, E. J., Perchuk, B. S., Skerker, J. M., Laub, M., 2012. Adaptive mutations that prevent crosstalk enable the expansion of paralogous signaling protein families. Cell 150, 222-232. 
Chamnongpol, S., Cromie, M., Groisman, E. A., 2003. $\mathrm{Mg}^{2+}$ sensing by the $\mathrm{Mg}^{2+}$ sensor PhoQ of Salmonella enterica. J. Mol. Biol. 325, 795-807.

Cherfils, J., Zeghouf, M., 2013. Regulation of small GTPases by GEFs, GAPs, and GDIs. Physiol Rev 93, 269-309.

Ciliberto, A., Capuani, F., Tyson, J. J., 2007. Modeling networks of coupled enzymatic reactions using the total quasi-steady state approximation. PLoS Comput. Biol. 3, e45.

Conradi, C., Flockerzi, D., Raisch, J., Stelling, J., 2007. Subnetwork analysis reveals dynamic features of complex (bio)chemical networks. Proc. Natl. Acad. Sci. USA 104, 19175-19180.

Cornish-Bowden, A., 2004. Fundamentals of Enzyme Kinetics, 3rd Edition. Portland Press, London.

Craciun, G., Tang, Y., Feinberg, M., 2006. Understanding bistability in complex enzyme-driven reaction networks. Proc. Natl. Acad. Sci. USA 103, $8697-$ 8702.

Dasgupta, T., Croll, D. H., Owen, J. A., Van der Heiden, M. G., Locasale, J. W., Alon, U., Cantley, L. C., Gunawardena, J., 2014. A fundamental trade-off in covalent switching and its circumvention by enzyme bifunctionality in glucose homeostasis. J Biol Chem 289, 13010-13025.

Dexter, J. P., Dasgupta, T., Gunawardena, J., 2015. Invariants reveal multiple forms of robustness in bifunctional enzyme systems. Integr. Biol. 7, 883894

Dexter, J. P., Gunawardena, J., 2013. Dimerization and bifunctionality confer robustness to the isocitrate dehydrogenase regulatory system in Escherichia Coli. J. Biol. Chem. 288, 5770-5778.

Dubey, B. N., Lori, C., Ozaki, S., Fucile, G., Plaza-Menacho, I., Jenal, U., Schirmer, T., 2016. Cyclic di-GMP mediates a histidine kianse/phosphatase switch by noncovalent domain cross-linking. Sci. Adv. 2, e1600823.

Dye, B. T., Schulman, B. A., 2007. Structural mechanisms underlying posttranslational modification by ubiquitin-like proteins. Annu. Rev. Biophys. Biomol. Struct. 36, 131-150.

Ehlert, F. J., 1988. Estimation of the affinities of allosteric ligands using radioligand binding and pharmacological null methods. Mol. Pharmacol. 33, 187 $-194$

Enserink, J. M., Kolodner, R. D., 2010. An overview of CDK1-controlled targets and processes. Cell Div 5, 11.

Estrada, J., Wong, F., DePace, A., Gunawardena, J., 2016. Information integration and energy expenditure in gene regulation. Cell 166, $234-244$.

Feliu, E., Knudsen, M., Andersen, L. N., Wiuf, C., 2012. An algebraic approach to signaling cascades with $n$ layers. Bull. Math. Biol. 74, 45-72.

Ferrell, Jr., J. E., 2002. Self-perpetuating states in signal transduction: positive feedback, double-negative feedback and bistability. Curr. Opin. Cell Biol. 14, 140-148.

Ferrell Jr., J. E., Ha, S. H., 2014. Ultrasensitivity part II: multisite phosphorylation, stoichiometric inhibitors, and positive feedback. Trends Biochem Sci 39, 556-569.

Fisher, S. L., Kim, S. K., Wanner, B. L., Walsh, C. T., 1996. Kinetic comparison of the specificity of the vancomycin kinase VanS for two response regulators, VanR and PhoB. Biochem. 35, 4732-4740.

Gao, R., Stock, A. M., 2013. Probing kinase and phosphatase activities of twocomponent systems in vivo with concentration-dependent phosphorylation profiling. Proc. Natl. Acad. Sci. USA 110, 672-677.

Garcia, E., Rhee, S. G., 1983. Cascade control of Escherichia coli glutamine synthetase. J. Biol. Chem. 258, 2246-2253.

Geontoro, L., Shoval, O., Kirschner, M. W., Alon, U., 2009. The incoherent feedforward loop can provide fold-change detection in gene regulation. Mol. Cell 36, 894-899.

Goldbeter, A., Koshland Jr., D. E., 1981. An amplified sensitivity arising from covalent modification in biological systems. Proc. Natl. Acad. Sci. USA 78, 6840-6844.

Gomez-Uribe, C., Verghese, G. C., Mirny, L. A., 2007. Operating regimes of signaling cycles: Statics, dynamics and noise filtering. PLoS Comput. Biol. $3, \mathrm{e} 246$.

Goody, R. S., Hofmann-Goody, W., 2002. Exchange factors, effectors, GAPs and motor proteins: common thermodynamic and kinetic principles for different functions. Eur Biophys J 31, 268-274.

Goryachev, A. B., Pokhilko, A. V., 2006. Computational model explains high activity and rapid cycling of Rho GTPases within protein complexes. PLoS Comput Biol 2, e172.

Goulian, M., 2010. Two-component signaling circuit structure and properties. Curr. Opin. Microbiol. 13, 184-189.
Grimshaw, C. E., Huang, S., Hanstein, C. G., Strauch, M. A., Burbulys, D., Wang, L., Hoch, J. A., Whiteley, J. M., 1998. Synergistic kinetic interactions between components of the phosphorelay controlling sporulation in Bacillus subtilis. Biochem. 37, 1365-1375.

Groban, E. S., Clarke, E. J., Salis, H. M., Miller, S. M., Voigt, C. A., 2009. Kinetic buffering of cross talk between bacterial two-component sensors. J. Mol. Biol. 390, 380-393.

Gu, C., Nguyen, H.-N., Hofer, A., Jessen, H. J., Dai, X., Wang, H., Shears, S. B., 2017. The significance of the bifunctional kinase/phosphatase activities of PPIP5Ks for coupling inositol pyrophosphate cell-signaling to cellular phosphate homeostasis. J. Biol. Chem. 292, 4544-4555.

Gunawardena, J., 2005. Multisite protein phosphorylation makes a good threshold but can be a poor switch. Proc. Natl. Acad. Sci. USA 102, 14617-14622.

Ha, S. H., Ferrell Jr., J. E., 2016. Thresholds and ultrasensitivity from negative cooperativity. Science 352, 990-993.

Ha, S. H., Kim, S. Y., Ferrell Jr., J. E., 2016. The prozone effect accounts for the paradoxical function of the Cdk-binding protein Suc1/Cks. Cell Rep. 14, 1408-1421.

Hart, Y., Madar, D., Yuan, J., Bren, A., Mayo, A. E., Rabinowitz, J. D., Alon, U., 2011a. Robust control of nitrogen assimilation by a bifunctional enzyme in E. coli. Mol. Cell 41, 117-127.

Hart, Y., Mayo, A. E., Milo, R., Alon, U., 2011b. Robust control of PEP formation rate in the carbon fixation pathway of $c_{4}$ plants by a bifunctional enzyme. BMC Syst Biol 5, 171.

Heermann, R., Jung, K., 2010. The complexity of the 'simple' two-component system $\mathrm{KdpD} / \mathrm{KdpE}$ in Escherichia coli. FEMS Microbiol. Lett. 304, 97 106

Heinrich, R., Neel, B. G., Rapoport, T. A., 2002. Mathematical models of protein kinase signal transduction. Mol. Cell 9, 957-970.

Igoshin, O. A., Alves, R., Savageau, M. A., 2008. Hysteretic and graded responses in bacterial two-component signal transduction. Mol. Microbiol. 68, 1196-1215.

Jiang, P., Ninfa, A. J., 1999. Regulator of autophosphorylation of Escherichia coli nitrogen regulator II by the PII signal transduction protein. J. Bact. 181, 1906-1911.

Jiang, P., Ventura, A. C., Ninfa, A. J., 2012. Characterization of the reconstituted UTase/UR-PII-NRII-NRI bicyclic signal transduction system that controls the transcription of nitrogen-regulated (Ntr) genes in Escherichia coli. Biochem. 51, 9045-9057.

Kim, S. Y., Ferrell, Jr., J. E., 2007. Substrate competition as a source of ultrasensitivity in the inactivation of Wee1. Cell 128, 1133-1145.

Kim, Y., Andreu, M. J., Lim, B., Chung, K., Terayama, M., Jiménez, G., Berg, C. A., Lu, H., Shvartsman, S. Y., 2011. Gene regulation by MAPK substrate competition. Dev Cell 20, 880-887.

Kim, Y., Coppey, M., Grossman, R., Ajuria, L., Jiménez, G., Paroush, Z., Shvartsman, S. Y., 2010. MAPK substrate competition integrates patterning signals in the Drosophilaembryo. Curr Biol 20, 446-451.

Krell, T., Lacal, J., Busch, A., Silva-Jiménez, H., Guazzaroni, M.-E., Ramos, J. L., 2010. Bacterial sensor kinases: Diversity in the recognition of environmental signals. Annu. Rev. Microbiol. 64, 539-559.

Kremling, A., Heermann, R., Centler, F., Jung, K., Gilles, E. D., 2004. Analysis of two-component signal transduction by mathematical modeling using the $K d p D / K d p E$ system of Escherichia Coli. Biosystems 78, 23-37.

Kurland, I. J., Pilkis, S. J., 1995. Covalent control of 6-phosphofructo-2kinase/fructose-2,6-bisphosphatase: insights into autoregulation of a bifunctional enzyme. Protein Sci. 4, 1023-1037.

Kuznetsov, Y. A., 1998. Elements of applied bifurcatuion theory. Springer Verlag, New York

LaPorte, D. C., Koshland Jr., D. E., 1982. A protein with kinase and phosphatase activities involved in regulation of tricarboxylic acid cycle. Nature 300, 458-460.

LaPorte, D. C., Koshland Jr., D. E., 1983. Phosphorylation of isocitrate dehydrogenase as a demonstration of enhanced sensitivity in covalent regulation. Nature 305, 286-290.

LaPorte, D. C., Thorsness, P. E., Koshland Jr., D. E., 1985. Compensatory phosphorylation of isocitrate dehydrogenase: A mechanism for adaption to the intracellular environment. J. Biol. Chem. 260, 10563-10568.

Laub, M. T., Goulian, M., 2007. Specificity in two-component signal transduction pathways. Annu Rev Genet 41, 121-145.

Legewie, S., Herzel, H., Westerhoff, H. V., Blüthgen, N., 2008. Recurrent design patterns in the feedback regulation of the mammalian signalling net- 
work. Mol. Syst. Biol. 4, 190.

Levine, E., Zhang, Z., Kuhlman, T., Hwa, T., 2007. Quantitative characteristics of gene regulation by small RNA. PLoS Biol 5, e229.

Levine, J. H., Lin, Y., Elowitz, M. B., 2013. Functional roles of pulsing in genetic circuits. Science 342, 1193-1200.

Lydeard, J. R., Schuman, B. A., Harper, J. W., 2013. Building an remodelling cullin-RING E3 ubiquitin ligases. EMBO Rep 14, 1050-1061.

Markevich, N. I., Hoek, J. B., Kholodenko, B. N., 2004. Signaling switches and bistability arising from multisite phosphorylation in protein kinase cascades. J. Cell Biol. 164, 353-359.

Masia, M. D., Garcia-Moreno, M., Garcia-Sevilla, F., Escribano, J., MolinaAlarcon, M., Amo-Saus, M. L., Ortiz-Ruiz, C. V., Varon, R., 2016. A novel transient phase kinetic analysis of the fractional modification of monocyclic enzyme cascades. J. Math. Chem. 54, 1952-1972.

Milo, R., Shen-Orr, S., Itzkovitz, S., Kashtan, N., Chklovskii, D., Alon, U., 2002. Network motifs: simple building blocks of complex networks. Science 298, 824-827.

Mitarai, N., Andersson, A. M. C., Krishna, S., Semsey, S., Sneppen, K., 2007. Efficient degradation and expression prioritization with small RNAs. Phys. Biol. 4, 164-171.

Miyashiro, T., Goulian, M., 2008. High stimulus unmasks positive feedback in autoregulated bacterial signaling circuit. Proc. Natl. Acad. Sci. USA 105, 17457-17462.

Mukherji, S., Ebert, M. S., Zheng, G. X. Y., Tsang, J. S., Sharp, P. A., van Oudenaarden, A., 2011. MicroRNAs can generate thresholds in target gene expression. Nat Genet 43, $854-859$.

Ninfa, A. J., Jiang, P., Atkinson, M. R., Peliska, J. A., 2000. Integration of antagonistic signals in the regulation of nitrogen assimilation in Escherichia coli. Curr. Top. Cell. Regul. 36, 31-75.

Novak, B., Tyson, J. J., 2008. Design principles of biochemical oscillators. Nat. Rev. Mol. Cell Biol. 9, 981-991.

Olsman, N., Geontoro, L., 2016. Allosteric proteins as logarithmic sensors. Proc. Natl. Acad. Sci. USA 113, 4423-4430.

Ortega, F., Acerenza, L., Westerhoff, H. V., Mas, F., Cascante, M., 2002. Product dependence and bifunctionality compromise the ultrasensitivity of signal transduction cascades. Proc. Natl. Acad. Sci. USA 99, 1170-1175.

Pedersen, M. G., Bersani, A. M., Bersami, E., Cortese, G., 2010. The total quasi-steady state approximation for complex enzyme reactions. J. Math. Biol. 60, 267-283.

Pedersen, M. G., Bersani, A. M., Bersani, E., 2007. The total quasi-steady state approximation for fully competitive enzyme reactions. Bull. Math. Biol. 69, 433-457.

Petroski, M. J., Deshaies, R. J., 2005. Function and regulation of cullin-ring ubiquitin ligases. Nat Rev Mol Cell Biol 6, 9-20.

Podgornaia, A. I., Laub, M. T., 2013. Determinants of specificity in twocomponent signal transduction. Curr. Opin. Microbiol. 16, 156 - 162.

Rosenfeld, N., Young, J. W., Alon, U., Swain, P. S., Elowitz, M. B., 2007. Accurate prediction of gene feedback circuit behavior from component properties. Mol. Syst. Biol. 3, 143.

Rowland, M. A., Deeds, E. J., 2014. Crosstalk and the evolution of specificity in two-component signaling. Proc Natl Acad Sci USA 111, 5550-5555.

Rowland, M. A., Fontana, W., Deeds, E. J., 2012. Crosstalk and competition in signaling networks. Biophys J 103, 2389-2398.

Russo, F. D., Silhavy, T. J., 1993. The essential tension: opposed reactions in bacterial two-component regulatory systems. Trends Microbiol 1, 306-310.

Salazar, C., Höfer, T., 2009. Multisite protein phosphorylation - from molecular mechanisms to kinetic models. FEBS J. 276, 3177-3198.

Sasagawa, S., Ozaki, Y., Fujita, K., Kuroda, S., 2005. Prediction and validation of the distinct dynamics of transient and sustained ERK activation. Nat. Cell Biol. 7, 365-373.

Sauro, H. M., Kholodenko, B. N., 2004. Quantitative analysis of signaling networks. Prog. Biophys. Mol. Biol. 86, 5-43.

Schnell, S., Maini, P. K., 2000. Enzyme kinetics at high enzyme concentrations. Bull. Math. Biol. 62, 483-499.

Segel, L. A., Slemrod, M., 1989. The quasi-steady state assumption: a case study in perturbation. SIAM Rev. 31, 446-477.

Shin, D., Lee, E. J., Huang, H., Groisman, E. A., 2006. A positive feedback loop promotes surge that jump-starts Salmonella virulence circuit. Science 314, 1607-1609.

Shinar, G., Feinberg, M., 2010. Structural sources of robustness in biochemical reaction networks. Science 327, 1389-1391.
Shinar, G., Milo, R., Martinez, M. R., Alon, U., 2007. Input-output robustness in simple bacterial signaling systems. Proc. Natl. Acad. Sci. USA 104, 19931-19935.

Shinar, G., Rabinowitz, J. D., Alon, U., 2009. Robustness in glyoxylate bypass regulation. PLoS Comput. Biol. 5, e1000297.

Siryaporn, A., Goulian, M., 2008. Cross-talk suppression between the CpxACpxR and EnvZ-OmpR two-component systems in E. coli. Mol. Microbiol. 70, 494-506.

Siryaporn, A., Perchuk, B. S., Laub, M. T., Goulian, M., 2010. Evolving a robust signal transduction pathway from weak cross-talk. Mol. Syst. Biol. 6, 452.

Skerker, J. M., Prasol, M. S., Perchuk, B. S., Biondi, E. G., Laub, M. T., 2005. Two-component signal transduction pathways regulating growth ad cell cycle progression in a bacterium: A system-level analysis. PLoS Biology 3, e334.

Sontag, E. D., 2017. Dynamic compensation, parameter identifiability, and equivariances. PLoS Comput. Biol. 13, e1005447.

Sourjik, V., 2004. Receptor clustering and signal processing in E. coli chemotaxis. Trends Microbiol 12, $569-576$.

Stewart, R. C., 2010. Protein histidine kinases: Assembly of active sites and their regulation in signaling pathways. Curr. Opin. Microbiol. 13, 133-141.

Stock, A. M., Robinson, V. L., Goudreau, P. N., 2000. Two-component signal transduction. Annu. Rev. Biochem. 69, 183-215.

Straube, R., 2012. Comment on 'load-induced modulation of signal transduction networks': Reconciling ultrasensitivity with bifunctionality? Sci. Signal. 5, lc1.

Straube, R., 2013. Sensitivity and robustness in covalent modification cycles with a bifunctional converter enzyme. Biophys J 105, 1925 - 1933.

Straube, R., 2014. Reciprocal regulation as a source of ultrasensitivity in twocomponent systems with a bifunctional sensor kinase. PLoS Comput Biol $10, \mathrm{e} 1003614$

Straube, R., 2015. Analysis of substrate competition in regulatory network motifs: Stimulus-response curves, thresholds and ultrasensitivity. J Theor Biol $380,74-82$.

Straube, R., 2017. Operating regimes of covalent modification cycles at high enzyme concentrations. J Theor Biol 431, 39-48.

Straube, R., Conradi, C., 2013. Reciprocal enzyme regulation as a source of bistability in covalent modification cycles. J. Theor. Biol. 330, 56-74.

Straube, R., Flockerzi, D., Wolf, D. A., 2017. Trade-off and flexibility in the dynamic regulation of the cullin-RING ubiquitin ligase repertoire. PLoS Comput Biol 10, e172.

Thomson, M., Gunawardena, J., 2009. Unlimited multistability in multisite phopshorylation systems. Nature 460, 274-277.

Thurley, K., Skupin, A., Thul, R., Falcke, M., 2012. Fundamental properties of $\mathrm{Ca}^{2+}$ signals. Biochim Biophys Acta 1820, 1185-1194.

Tindall, M. J., Porter, S. L., Maini, P. K., Armitage, J. P., 2013. Modeling chemotaxis reveals the role of reversed phosphotransfer and bi-functional kinase-phosphatase. PLoS Comput. Biol. 6, e1000896.

Tiwari, A., Ray, J. C. J., Narula, J., Igoshin, O. A., 2011. Bistable responses in bacterial genetic networks: Designs and dynamical consequences. Math. Biosci. 231, 76-89.

Tzafriri, A. R., 2003. Michaelis-Menten kinetics at high enzyme concentrations. Bull. Math. Biol. 65, 1111-1129.

Tzafriri, A. R., Edelman, E. R., 2004. The total quasi-steady state approximation is valid for reversible enzyme kinetics. J. Theor. Biol. 226, 303-313.

Ubersax, J. A., Woodbury, E. L., Quang, P. N., Paraz, M., Blethrow, J. D., Shah, K., Shokat, K. M., Morgan, D. O., 2003. Targets of the cyclin-dependent kinase cdk1. Nature 425, 859-864.

Ventura, A. C., Jiang, P., Van Wassenhove, L., Del Vecchio, D., Merajver, S. D., Ninfa, A. J., 2010. Signaling properties of a covalent modification cycle are altered by a downstream target. Proc. Natl. Acad. Sci. USA 107, 1003210037.

Wei, K., Moinat, M., Maarleveld, T. R., Bruggeman, F. J., 2014. Stochastic simulation of procaryotic two-component signalling indicates stochasticityinduced active-state locking and growth-rate dependent bistability. Mol. Biosyst. 10, 2338 - 2346.

Witzel, F., Maddison, L., Blüthgen, N., 2012. How scaffolds shape MAPK signaling: what we know and opportunities for systems approaches. Front Physiol 3, 475.

Zheng, J., Jia, Z., 2010. Structure of the bifunctional isocitrate dehydrogenase kinase/phosphatase. Nature 465, 961-965. 


\section{Supporting Information for}

Analysis of Network Motifs in Cellular Regulation

Ronny Straube 


\section{Cross-talk in two-component systems}

The phosphorylation of two response regulators (RRs) by a single sensor kinase (HK), as depicted in Fig. 15C, is described by the reaction mechanism

$$
\begin{aligned}
H K^{*}+R R_{i} \underset{k_{t, i}^{-}}{\stackrel{k_{t, i}^{+}}{\rightleftarrows}} H K^{*}-R R_{i} \stackrel{k_{t, i}}{\rightarrow} H K+R R_{i}^{*}, \quad i=1,2 \\
H K+R R_{i}^{*} \underset{k_{p, i}^{+}}{\stackrel{k_{p, i}^{+}}{\rightleftarrows}} H K-R R_{i}^{*} \stackrel{k_{p, i}}{\rightarrow} H K+R R_{i}, \quad i=1,2 \\
H K \underset{k_{K}^{-}}{\stackrel{k_{K}^{+}}{\rightleftarrows}} H K^{*}
\end{aligned}
$$

where $H K^{*}$ and $R R_{i}^{*}$ denote the phosphorylated forms of the sensor kinase and the response regulator, respectively. The corresponding ODE system reads

$$
\begin{aligned}
\frac{d\left[R R_{i}^{*}\right]}{d t}= & k_{t, i}\left[H K^{*}-R R_{i}\right]-k_{p, i}^{+}\left[R R_{i}^{*}\right][H K]+k_{p, i}^{-}\left[H K-R R_{i}^{*}\right] \\
\frac{d[H K]}{d t}= & -k_{K}^{+}[H K]+k_{K}^{-}\left[H K^{*}\right]+\sum_{i=1}^{2} k_{t, i}\left[H K^{*}-R R_{i}\right] \\
& -\sum_{i=1}^{2}\left(k_{p, i}^{+}\left[R R_{i}^{*}\right][H K]-\left(k_{p, i}^{-}+k_{p, i}\right)\left[H K-R R_{i}^{*}\right]\right) \\
\frac{d\left[H K^{*}-R R_{i}\right]}{d t}= & k_{t, i}^{+}\left[R R_{i}\right]\left[H K^{*}\right]-\left(k_{t, 1}^{-}+k_{t, i}\right)\left[H K^{*}-R R_{i}\right] \\
\frac{d\left[H K-R R_{i}^{*}\right]}{d t}= & k_{p, i}^{+}\left[R R_{i}^{*}\right][H K]-\left(k_{p, i}^{-}+k_{p, i}\right)\left[H K-R R_{i}^{*}\right]
\end{aligned}
$$

where $\left[R R_{1}\right],\left[R R_{2}\right]$ and $\left[H K^{P}\right]$ have to be replaced according to the conservation relations

$$
\begin{aligned}
{\left[R R_{1}\right]+\left[R R_{1}^{*}\right]+\left[H K^{*}-R R_{1}\right]+\left[H K-R R_{1}^{*}\right] } & =R R_{1 T} \\
{\left[R R_{2}\right]+\left[R R_{2}^{*}\right]+\left[H K^{*}-R R_{2}\right]+\left[H K-R R_{2}^{*}\right] } & =R R_{2 T} \\
{[H K]+\left[H K^{*}\right]+\sum_{i=1}^{2}\left[H K^{*}-R R_{i}\right]+\sum_{i=1}^{2}\left[H K-R R_{i}^{*}\right] } & =H K_{T} .
\end{aligned}
$$

We assume that both RRs are in excess so that

$$
\begin{aligned}
& {\left[R R_{1}\right] \approx R R_{1 T}-\left[R R_{1}^{*}\right]} \\
& {\left[R R_{2}\right] \approx R R_{2 T}-\left[R R_{2}^{*}\right]}
\end{aligned}
$$


Under steady state conditions the enzyme-substrate complexes are given by

$$
\begin{aligned}
{\left[H K^{*}-R R_{i}\right] } & =\frac{\left[R R_{i}\right]\left[H K^{*}\right]}{K_{t, i}} \approx \frac{\left(R R_{i T}-\left[R R_{i}^{*}\right]\right)\left[H K^{*}\right]}{K_{t, i}} \\
{\left[H K-R R_{i}^{*}\right] } & =\frac{\left[R R_{i}^{*}\right][H K]}{K_{p, i}}
\end{aligned}
$$

where the Michaelis-Menten constants are defined by

$$
K_{t, i}=\frac{k_{t, i}+k_{t, i}^{-}}{k_{t, i}^{+}} \quad \text { and } \quad K_{p, i}=\frac{k_{p, i}+k_{p, i}^{-}}{k_{p, i}^{+}}, \quad i=1,2 .
$$

Addition of Eqs. (S2) and (S4) yields at steady state

$$
\begin{aligned}
{[H K] } & =\frac{k_{K}^{-}}{k_{K}^{+}}\left[H K^{*}\right]+\frac{k_{t, 1}}{k_{K}^{+}}\left[H K^{*}-R R_{1}\right]+\frac{k_{t, 2}}{k_{K}^{+}}\left[H K^{*}-R R_{2}\right] \\
& =\frac{k_{K}^{-}}{k_{K}^{+}}\left(1+\frac{k_{t, 1}}{k_{K}^{-}} \frac{\left[R R_{1}\right]}{K_{t, 1}}+\frac{k_{t, 2}}{k_{K}^{-}} \frac{\left[R R_{2}\right]}{K_{t, 2}}\right)\left[H K^{*}\right] \\
& \approx \frac{k_{K}^{-}}{k_{K}^{+}}\left(1+\frac{k_{t, 1}^{-}}{k_{K}^{-}} \frac{R R_{1 T}-\left[R R_{1}^{*}\right]}{K_{t, 1}}+\frac{k_{t, 2}}{k_{K}^{-}} \frac{R R_{2 T}-\left[R R_{2}^{*}\right]}{K_{t, 2}}\right)\left[H K^{*}\right]
\end{aligned}
$$

where we have used the conservation relations Eqs. (S8) in the last line. Similarly, addition of Eqs. (S1) and (S4) yields the steady state relations

$$
\begin{aligned}
k_{t, 1}\left[H K^{*}-R R_{1}\right] & =k_{p, 1}\left[H K-R R_{1}^{*}\right] \\
k_{t, 2}\left[H K^{*}-R R_{2}\right] & =k_{p, 2}\left[H K-R R_{2}^{*}\right] .
\end{aligned}
$$

Replacing the enzyme-substrate complexes by the relations in Eqs. (S9) and the conservation relations Eqs. (S8) yields

$$
\begin{aligned}
k_{t, 1} \frac{\left(R R_{1 T}-\left[R R_{1}^{*}\right]\right)\left[H K^{*}\right]}{K_{t, 1}} & \approx k_{p, 1} \frac{\left[R R_{1}^{*}\right][H K]}{K_{p, 1}} \\
k_{t, 2} \frac{\left(R R_{2 T}-\left[R R_{2}^{*}\right]\right)\left[H K^{*}\right]}{K_{t, 2}} & \approx k_{p, 2} \frac{\left[R R_{2}^{*}\right][H K]}{K_{p, 2}} .
\end{aligned}
$$

Finally, replacing $[H K]$ on the right-hand sides by the expression in Eq. (S10) the factor $\left[H K^{*}\right]$ cancels on both sides of the equation resulting in the steady state equations

$$
\begin{aligned}
k_{t, 1} \frac{\left(R R_{1 T}-\left[R R_{1}^{*}\right]\right)}{K_{t, 1}} & \approx k_{p, 1} \frac{\left[R R_{1}^{*}\right]}{K_{p, 1}} \frac{k_{K}^{-}}{k_{K}^{+}}\left(1+\frac{k_{t, 1}}{k_{K}^{-}} \frac{R R_{1 T}-\left[R R_{1}^{*}\right]}{K_{t, 1}}+\frac{k_{t, 2}}{k_{K}^{-}} \frac{R R_{2 T}-\left[R R_{2}^{*}\right]}{K_{t, 2}}\right) \\
k_{t, 2} \frac{\left(R R_{2 T}-\left[R R_{2}^{*}\right]\right)}{K_{t, 2}} & \approx k_{p, 2} \frac{\left[R R_{2}^{*}\right]}{K_{p, 2}} \frac{k_{K}^{-}}{k_{K}^{+}}\left(1+\frac{k_{t, 1}}{k_{K}^{-}} \frac{R R_{1 T}-\left[R R_{1}^{*}\right]}{K_{t, 1}}+\frac{k_{t, 2}}{k_{K}^{-}} \frac{R R_{2 T}-\left[R R_{2}^{*}\right]}{K_{t, 2}}\right) .
\end{aligned}
$$


By defining the rescaled Michaelis-Menten constants $C_{p, i}$ and $C_{t, i}$ through

$$
C_{p, i}=\frac{k_{K}^{+}}{k_{p, i}} K_{p, i} \quad \text { and } C_{t, i}=\frac{k_{K}^{-}}{k_{t, i}} K_{t, i}, \quad i=1,2
$$

Eqs. (S11) can be written in the form

$$
\begin{aligned}
\frac{\left(R R_{1 T}-\left[R R_{1}^{*}\right]\right)}{C_{t, 1}} & \approx \frac{\left[R R_{1}^{*}\right]}{C_{p, 1}}\left(1+\frac{R R_{1 T}-\left[R R_{1}^{*}\right]}{C_{t, 1}}+\frac{R R_{2 T}-\left[R R_{2}^{*}\right]}{C_{t, 2}}\right) \\
\frac{\left(R R_{2 T}-\left[R R_{2}^{*}\right]\right)}{C_{t, 2}} & \approx \frac{\left[R R_{2}^{*}\right]}{C_{p, 2}}\left(1+\frac{R R_{1 T}-\left[R R_{1}^{*}\right]}{C_{t, 1}}+\frac{R R_{2 T}-\left[R R_{2}^{*}\right]}{C_{t, 2}}\right) .
\end{aligned}
$$

Taking the ratio of both equations yields

$$
\frac{C_{p, 1}}{C_{t, 1}} \frac{\left(R R_{1 T}-\left[R R_{1}^{*}\right]\right)}{\left[R R_{1}^{*}\right]}=\frac{C_{p, 2}}{C_{t, 2}} \frac{\left(R R_{2 T}-\left[R R_{2}^{*}\right]\right)}{\left[R R_{2}^{*}\right]}
$$

or

$$
\left[R R_{2}^{*}\right]=\frac{R_{2 T}\left[R R_{1}^{*}\right]}{\frac{\varepsilon_{p}}{\varepsilon_{t}}\left(R_{1 T}-\left[R R_{1}^{*}\right]\right)+\left[R R_{1}^{*}\right]}
$$

where

$$
\varepsilon_{p} \equiv \frac{C_{p, 1}}{C_{p, 2}}=\frac{k_{p, 2} / K_{p, 2}}{k_{p, 1} / K_{p, 1}} \quad \text { and } \quad \varepsilon_{t} \equiv \frac{C_{t, 1}}{C_{t, 2}}=\frac{k_{t, 2} / K_{t, 2}}{k_{t, 1} / K_{t, 1}}
$$

denote the ratios of the kinetic preferences of the HK's phosphatase activity $\left(\varepsilon_{p}\right)$ and the HK's phosphotransferase activity $\left(\varepsilon_{t}\right)$ with respect to the two RRs.

Substituting the relation

$$
R_{2 T}-\left[R R_{2}^{*}\right]=\frac{R_{2 T} \frac{\varepsilon_{p}}{\varepsilon_{t}}\left(R_{1 T}-\left[R R_{1}^{*}\right]\right)}{\frac{\varepsilon_{p}}{\varepsilon_{t}}\left(R_{1 T}-\left[R R_{1}^{*}\right]\right)+\left[R R_{1}^{*}\right]}
$$

into Eq. (S12) yields a cubic equation for $\left[R R_{1}^{*}\right]$ which can be written as

$$
\begin{aligned}
\left(1-\frac{\varepsilon_{p}}{\varepsilon_{t}}\right)\left[R R_{1}^{*}\right]^{3}-\left(R R_{1 T}+C_{p, 1}-\varepsilon_{p} R R_{2 T}+C_{t, 1}-\frac{\varepsilon_{p}}{\varepsilon_{t}}\left(2 \cdot R R_{1 T}+C_{p, 1}+C_{t, 1}\right)\right)\left[R R_{1}^{*}\right]^{2} & (\mathrm{~S} 15) \\
+ & R R_{1 T}\left(C_{p, 1}-\varepsilon_{p} R R_{2 T}-\frac{\varepsilon_{p}}{\varepsilon_{t}}\left(R R_{1 T}+2 C_{p, 1}+C_{t, 1}\right)\right)\left[R R_{1}^{*}\right]+\frac{\varepsilon_{p}}{\varepsilon_{t}} C_{p, 1} R R_{1 T}^{2}=0
\end{aligned}
$$

By symmetry the steady state equation for $\left[R R_{2}^{*}\right]$ is given by

$$
\begin{aligned}
\left(1-\frac{\varepsilon_{t}}{\varepsilon_{p}}\right)\left[R R_{2}^{*}\right]^{3}-\left(R R_{2 T}+C_{p, 2}-\frac{1}{\varepsilon_{p}} R R_{1 T}+C_{t, 2}-\frac{\varepsilon_{t}}{\varepsilon_{p}}\left(2 \cdot R R_{2 T}+C_{p, 2}+C_{t, 2}\right)\right)\left[R R_{2}^{*}\right]^{2} & (\mathrm{~S} 16) \\
+ & R R_{2 T}\left(C_{p, 2}-\frac{1}{\varepsilon_{p}} R R_{1 T}-\frac{\varepsilon_{t}}{\varepsilon_{p}}\left(R R_{2 T}+2 C_{p, 2}+C_{t, 2}\right)\right)\left[R R_{2}^{*}\right]+\frac{\varepsilon_{t}}{\varepsilon_{p}} C_{p, 2} R R_{2 T}^{2}=0
\end{aligned}
$$


Note that these equations are structurally identical with that for the receptor-ligand complex arising in the competition of two ligands for a receptor binding site in Eq. (35) if one makes the substitutions (cf. Table 1)

$$
\begin{aligned}
{\left[R R_{1}^{*}\right] } & \leftrightarrow[L 1 . R], \quad R R_{1 T} \leftrightarrow L 1_{T}, \quad \frac{\varepsilon_{p}}{\varepsilon_{t}} \leftrightarrow \varepsilon \\
C_{p, 1} & \leftrightarrow R_{T}, \quad \varepsilon_{p} R R_{2 T} \leftrightarrow L 2_{T}, \quad C_{t, 1} \leftrightarrow K_{d 1}
\end{aligned}
$$

in the case of Eq. (S15) and

$$
\begin{aligned}
{\left[R R_{2}^{*}\right] } & \leftrightarrow[L 1 . R], \quad R R_{2 T} \leftrightarrow L 1_{T}, \quad \frac{\varepsilon_{t}}{\varepsilon_{p}} \leftrightarrow \varepsilon \\
C_{p, 2} & \leftrightarrow R_{T}, \quad \frac{1}{\varepsilon_{p}} R R_{1 T} \leftrightarrow L 2_{T}, \quad C_{t, 2} \leftrightarrow K_{d 1}
\end{aligned}
$$

in the case of Eq. (S16).

In the limit $\varepsilon_{p} \rightarrow \infty$ (with $\varepsilon_{t}$ constant) the dominant terms in Eqs. (S15) are

$$
\begin{gathered}
-\frac{\varepsilon_{p}}{\varepsilon_{t}}\left[R R_{1}^{*}\right]^{3}+\left(\varepsilon_{p} R R_{2 T}+\frac{\varepsilon_{p}}{\varepsilon_{t}}\left(2 \cdot R R_{1 T}+C_{p, 1}+C_{t, 1}\right)\right)\left[R R_{1}^{*}\right]^{2} \\
+R R_{1 T}\left(\varepsilon_{p} R R_{2 T}+\frac{\varepsilon_{p}}{\varepsilon_{t}}\left(2 C_{p, 1}+R R_{1 T}+C_{t, 1}\right)\right)\left[R R_{1}^{*}\right]+\frac{\varepsilon_{p}}{\varepsilon_{t}} C_{p, 1} R R_{1 T}^{2} \approx 0
\end{gathered}
$$

which can be factorized as

$$
\left(\left[R R_{1}^{*}\right]-R R_{1 T}\right)\left(\left[R R_{1}^{*}\right]^{2}-\left(R R_{1 T}+C_{p, 1}+C_{t, 1}+\varepsilon_{t} R R_{2 T}\right)\left[R R_{1}^{*}\right]+C_{p, 1} R R_{1 T}\right) \approx 0 .
$$

Hence, $\left[R R_{1}^{*}\right] \approx R_{1 T}$ or $\left[R R_{1}^{*}\right]$ is a solution of the $\mathbf{L R}$-type equation

$$
\left[R R_{1}^{*}\right]^{2}-\left(R R_{1 T}+C_{p, 1}+C_{t, 1}+\varepsilon_{t} R R_{2 T}\right)\left[R R_{1}^{*}\right]+C_{p, 1} R R_{1 T} \approx 0 .
$$

In contrast, in the limit $\varepsilon_{t} \rightarrow \infty$ the dominant terms of Eq. (S15) are given by

$$
\left(\left[R R_{1}^{*}\right]^{2}-\left(C_{t, 1}+R R_{1 T}+C_{p, 1}-\varepsilon_{p} R R_{2 T}\right)\left[R R_{1}^{*}\right]+R R_{1 T}\left(C_{p, 1}-\varepsilon_{p} R R_{2 T}\right)\right)\left[R R_{1}^{*}\right] \approx 0 .
$$

Hence, $\left[R R_{1}^{*}\right] \approx 0$ or $\left[R R_{1}^{*}\right]$ is a solution of the $\mathbf{L R}$-type equation

$$
\left[R R_{1}^{*}\right]^{2}-\left(R R_{1 T}+C_{p, 1}-\varepsilon_{p} R R_{2 T}+C_{t, 1}\right)\left[R R_{1}^{*}\right]+\left(C_{p, 1}-\varepsilon_{p} R R_{2 T}\right) R R_{1 T} \approx 0 .
$$

Note that similar as for Eq. (38) the solution of Eq. (S18) is only defined for $C_{p, 1}>\varepsilon_{p} R R_{2 T}$ or $k_{K}^{+}>\left(k_{p, 2} / K_{p, 2}\right) R R_{2 T}$. In the opposite case $\left(k_{K}^{+}<\left(k_{p, 2} / K_{p, 2}\right) R R_{2 T}\right)$ the approximation can be obtained by substituting the corresponding quantities (S17) into Eq. (37) which yields

$$
\left[R R_{1}^{*}\right] \approx \frac{1}{\varepsilon_{t}} \frac{R R_{1 T} C_{p, 1}}{R R_{2 T}-C_{p, 2}}, \quad k_{K}^{+}<\frac{k_{p, 2}}{K_{p, 2}} R R_{2 T} .
$$


For $\left[R R_{2}^{*}\right]$ as described by Eq. (S16) the dominant terms are can be factorized as

$$
\left[R R_{2}^{*}\right]\left(\left[R R_{2}^{*}\right]^{2}-\left(R R_{2 T}+C_{p, 2}+C_{t, 2}\right)\left[R R_{2}^{*}\right]+R R_{2 T} C_{p, 2}\right) \approx 0
$$

if $\varepsilon_{t} \rightarrow \infty$ and

$$
\left(R R_{2 T}-\left[R R_{2}^{*}\right]\right)\left(\left[R R_{2}^{*}\right]^{2}-\left[R R_{2}^{*}\right]\left(R R_{2 T}+C_{p, 2}+C_{t, 2}\right)+R R_{2 T} C_{p, 2}\right) \approx 0 .
$$

if $\varepsilon_{p} \rightarrow \infty$. Hence, in either limit the steady state of $\left[R R_{2}^{*}\right]$ is determined by the same quadratic equation which is identical with that of the Batchelor-Goulian model for a single RR (cf. Eq. 61). 